Article

\title{
Real Time Information Based Energy Management Using Customer Preferences and Dynamic Pricing in Smart Homes
}

\author{
Muhammad Babar Rasheed ${ }^{1}$, Nadeem Javaid ${ }^{1, *}$, Muhammad Awais ${ }^{2}$, Zahoor Ali Khan ${ }^{3}$, \\ Umar Qasim ${ }^{4}$, Nabil Alrajeh ${ }^{5}$, Zafar Iqbal ${ }^{6}$ and Qaisar Javaid ${ }^{7}$ \\ 1 COMSATS Institute of Information Technology, Islamabad 44000, Pakistan; babar_mehr@yahoo.com \\ 2 Department of Technology, The University of Lahore, Lahore 54000, Pakistan; \\ m.awais.qureshi27@gmail.com \\ 3 Internetworking Program, Faculty of Engineering, Dalhousie University, Halifax, NS B3J 4R2, Canada; \\ zahoor.khan@dal.ca \\ 4 Cameron Library, University of Alberta, Edmonton, AB T6G 2J8, Canada; umar.qasim@ualberta.ca \\ 5 Department of Biomedical Technology, College of Applied Medical Sciences, King Saud University, \\ Riyadh 11633, Saudi Arabia; nabilksu@yahoo.com \\ 6 University Institute of Information Technology, Pir Mehr Ali Shah Arid Agriculture University, \\ Rawalpindi 46000, Pakistan; iqbal.zafar9@gmail.com \\ 7 Department of Computer Science \& Software Engineering, International Islamic University, \\ Islamabad 44000, Pakistan; qaisar@iiu.edu.pk \\ * Correspondence: nadeemjavaidqau@gmail.com; Tel.: +92-300-579-2728
}

Academic Editor: G.J.M. (Gerard) Smit

Received: 10 March 2016; Accepted: 5 July 2016; Published: 14 July 2016

\begin{abstract}
This paper presents real time information based energy management algorithms to reduce electricity cost and peak to average ratio (PAR) while preserving user comfort in a smart home. We categorize household appliances into thermostatically controlled $(t c)$, user aware $(u a)$, elastic $(e l)$, inelastic (iel) and regular $(r)$ appliances/loads. An optimization problem is formulated to reduce electricity cost by determining the optimal use of household appliances. The operational schedules of these appliances are optimized in response to the electricity price signals and customer preferences to maximize electricity cost saving and user comfort while minimizing curtailed energy. Mathematical optimization models of $t c$ appliances, i.e., air-conditioner and refrigerator, are proposed which are solved by using intelligent programmable communication thermostat ( iPCT). We add extra intelligence to conventional programmable communication thermostat (CPCT) by using genetic algorithm (GA) to control tc appliances under comfort constraints. The optimization models for $u a, e l$, and iel appliances are solved subject to electricity cost minimization and PAR reduction. Considering user comfort, $e l$ appliances are considered where users can adjust appliance waiting time to increase or decrease their comfort level. Furthermore, energy demand of $r$ appliances is fulfilled via local supply where the major objective is to reduce the fuel cost of various generators by proper scheduling. Simulation results show that the proposed algorithms efficiently schedule the energy demand of all types of appliances by considering identified constraints (i.e., PAR, variable prices, temperature, capacity limit and waiting time).
\end{abstract}

Keywords: demand side management; optimization; energy management; real time pricing; genetic algorithm (GA); knapsack; smart grid (SG); programmable communication thermostat; microgird

\section{Introduction}

With the rapid increase in the world's population, electricity demand also increases. It is estimated that total energy demand at the end of 2020 will increase by $75 \%$ as compared to 2000 [1]. 
This increase may force utilities to rethink electricity generation and distribution in order to avoid unprecedented energy challenges. The utilities thus struggle to fulfill and manage the energy demand with smart generation with reduced carbon emissions. For this purpose, the traditional electric grid is evolving to a new smart grid (SG) [2]. In SG, advanced information and communication technologies provide flexibility to interact customers with utility [3,4]. Advanced metering infrastructure (AMI) equips each customer with smart meter whose major function is to gather energy demand information at customer premises and upload to the utility server [5]. According to [6,7], SG allows integration of renewable and distributed energy generation to diminish the effects of $\mathrm{CO}_{2}$ on environment and to reduce the energy consumption.

Demand side management (DSM) is one of the key programs of SG to efficiently manage the energy demand of end users via real time information exchange between utility and consumer through AMI. These programs aim at enhancing grid reliability by reducing average peak load demand. So, utilities and customers can manage the energy generation and consumption through the implementation of DSM programs by providing incentives or encouraging the customers to participate in energy management programs. End users can take monitory benefits by shifting peak load during off peak hours by adopting different scheduling techniques.

In early DSM programs, utilities manually shed the selected load of consumers during critical peak hours. On the other hand, load shifting in SG is realized by encouraging customers towards shifting their load to avoid peak to average ratio (PAR) $[8,9]$. Although shifting the load from high peak to low peak hours reduces the peak load and electricity cost saving, it may decrease user comfort. So, user comfort maximization and electricity cost minimization are two contradictory objectives to be achieved at the same time. Thus, mechanisms are needed that takes into consideration user preferences, energy price signals, and other comfort constraints. Moreover, these types of mechanisms must be capable enough to handle uncertainties in customer preferences and price variations.

Besides considering end user preferences and needs, utilities must also provide high incentives to motivate customers to reschedule their load to flatten high peaks. Such challenges have motivated the need for intelligent energy management algorithms that can handle all types of loads and responds to price variations. To address these challenges, this paper presents energy management algorithms optimize the energy consumption schedules of household appliances while meeting constraints. The major contributions of the proposed work are given as follows:

(1) We propose an algorithm to control energy consumption and electricity cost of heating ventilation and air conditioning (HVAC) system while considering dynamic pricing and user comfort constraints.

(2) We propose an algorithm to control the energy consumption of a refrigerator by considering thermal and other constraints.

(3) We propose an algorithm to control the working of various household appliances (e.g., fans, and lights) using WSNs to minimize the energy consumption.

(4) For $r$ appliances, a microgrid model for local electricity generation where different generation units are considered is proposed and their duty cycles are formulated according to the energy demand.

(5) We propose a simple model to show that user comfort is directly related to the waiting time of appliances. Here, the appliance waiting time is directly related to the appliances having flexible starting time requirements $(e l, i e l)$.

(6) For electricity cost and PAR reduction, knapsack problem formulation technique is used which later on is solved by using genetic algorithm (GA) algorithm.

(7) To analyze the effectiveness of the proposed algorithms, we perform simulations and compare the results with scheduled and unscheduled cases.

The rest of this paper is organized as follows. In Section 2, related work is discussed. In Section 3, system model and appliance classification model are discussed, respectively. Section 4 discusses the 
proposed algorithms. Simulation results are discussed in Section 5. Section 6 gives the conclusion. The variables used in this work are given in Table 1.

Table 1. Nomenclature. HVAC: heating ventilation and air conditioning.

\begin{tabular}{|c|c|c|c|}
\hline Symbol & Description & Symbol & Description \\
\hline tc & Thermostatically controlled appliances & $t n$ & Total time interval \\
\hline ua & User aware appliances & $F()$. & Fitness function \\
\hline el & Elastic appliances & $\varsigma$ & Electricity cost \\
\hline iel & Inelastic appliances & $\varphi$ & Appliance on/off status \\
\hline$r$ & Regular appliances & $t_{\min }, t_{\max }$ & Minimum and maximum time limits \\
\hline$n$ & Total number of appliances & $\alpha$ & Total number of occupants \\
\hline$\iota_{i}$ & Cost due to losses & $e_{\mathrm{S}}$ & Standby energy consumption of refrigerator \\
\hline$C_{t}$ & Total electricity cost & $e_{\mathrm{c}}$ & Energy consumption of compressor \\
\hline$b_{i}$ & Total fuel cost & $e_{\mathrm{i}_{\mathrm{do}}}$ & Energy consumption increment with door open \\
\hline$u$ & Total number of generators & $e_{t_{\text {in }}}$ & Energy consumption increment with temperature \\
\hline$c_{i}$ & Generator startup cost & $t e_{r}^{\max }$ & Maximum room temperature \\
\hline$P_{i}$ & Power produced by generator & $D^{\mathrm{m}}$ & Maximum delay \\
\hline$P^{\max }$ & Maximum power & $\beta$ & Total number of times the door is open \\
\hline$P^{\min }$ & Minimum power & $\gamma$ & Change in outdoor temperature \\
\hline$E^{r}$ & Total energy consumption of $r$ appliances & $t e_{\text {ref }}$ & Internal temperature of refrigerator \\
\hline$E^{t c}$ & Total energy consumption of $t c$ appliances & $t e_{\mathrm{ref}}^{\max }$ & Refrigerator's maximum internal temperature \\
\hline$E^{e l}$ & Total energy consumption of $e l$ appliances & $t e_{\mathrm{ref}}^{\mathrm{mer}}$ & Refrigerator's minimum internal temperature \\
\hline$E^{i e l}$ & Total energy consumption of iel appliances & $e_{\mathrm{f}}$ & Energy consumption of a fan \\
\hline$E^{u a}$ & Total energy consumption of $u a$ appliances & $e_{1}$ & Energy consumption of a light \\
\hline$E^{h}$ & Total energy consumption of HVAC & $t e_{\text {in }}$ & Indoor temperature \\
\hline$D^{a}$ & Average delay & $t e_{\mathrm{ot}}$ & outdoor temperature \\
\hline$t^{\mathrm{sch}}$ & Appliance scheduled start time & $\delta_{l}$ & Occupancy sensor at left side of room \\
\hline$p_{\mathrm{m}}$ & Maximum electricity peaks & $\delta_{r}$ & Occupancy sensor at right side of room \\
\hline$\Phi$ & Status of light flag $[0,1]$ & $t^{\mathrm{u}-\mathrm{sch}}$ & Appliance unscheduled start time \\
\hline$\lambda$ & Average user frustration & $t_{l_{i}}$ & Length of operation time \\
\hline$\theta$ & Power capacity limit & $e_{\bullet}^{h}$ & Energy consumption of HVAC with occupants \\
\hline & Average electricity peaks & $e_{\circ}^{h}$ & Energy consumption of HVAC without occupants \\
\hline$t e_{r}^{\min }$ & Minimum room temperature & $\tau$ & Appliance scheduling horizon \\
\hline
\end{tabular}

\section{Related Work}

A linear programming based technique for residential energy management is proposed in [10]. This approach efficiently reduces the electricity cost and PAR. Moreover, user comfort is also increased by reducing appliance waiting time, however, price flexibility is not taken into account. In [11], linear programming technique is used to solve residential energy management problem. This approach is efficient in terms of electricity cost reduction, however, more computational power is required to solve complex optimization problem. Moreover, linear programming based optimization technique only solve the load having linear characteristics. In [12], authors use a monotonic optimization techniques to solve appliance scheduling problem. In this technique, renewable energy is used along with grid energy to fulfill energy demand during critical peak hours. Although, renewable energy is beneficial in reducing PAR and electricity cost however, this increases the integration complexity in the system. Considering variable loads, a mixed integer linear programming approach is used for cost efficient scheduling [13]. In general, these techniques are efficient in reducing electricity cost of the end users along with PAR reduction. However, these techniques are computationally expensive and user comfort is not modeled.

On the other hand, heuristic optimization techniques are widely used to solve energy optimization problems in SG. These techniques are efficient in solving both linear and nonlinear energy optimization problems. In [14], an optimal stoping rule based technique is used to schedule appliances. The authors model user comfort in terms of appliance waiting time cost. This technique works on different thresholds defined by users, and these thresholds are measured when electricity price signal is uniformly distributed. The scheme is efficient in real time price (RTP) environment and for limited number of appliances. In [15], the authors categorized appliances on the bases of 
user comfort and electricity cost reduction. Mathematical models for each class of appliances are proposed where electricity cost reduction and user comfort are considered as a joint problem which is solved by using wind driven optimization (WDO) algorithm. For PAR and electricity cost reduction, Knapsack-wind driven optimization (K-WDO) is also proposed. Although, the proposed techniques are efficient in handling user comfort and electricity cost reduction. However, $(t c)$ appliances such as air-conditioner, and other user dependant appliances are not considered because inconsideration of thermal and occupancy constraints makes the working of these appliances are difficult to optimize.

Due to the unpredictable nature of distributed renewable energy sources and loads results non-linear problem in a large scale. To manage the operational cost of power plant, particle swan optimization (PSO) algorithm has been used in [16]. In [17], a heuristic based evolutionary algorithm (EA) has been used to schedule a large number of home appliances to reduce electricity cost and high peaks. Three service areas including residential, commercial and industrial are considered to perform simulations. Based upon real time pricing, appliance scheduling for cost reduction objective is performed using stochastic and robust optimization techniques [18]. On the bases of energy demand and temporal characteristics, the appliances are categorized into deferrable, non-deferrable, interruptible and non-interruptible ones. These techniques [16-18] are efficient in reducing electricity cost. However, user comfort is neglected which is major objective of the proposed work. In [7,19], both linear programming and game theoretic techniques for centralized and distributed energy management are proposed. PAR reduction and its effects on generation efficiency are also analyzed.

In [20], authors propose a load control technique for multiple residential units where utility company use a cost function to provide electricity to end users. Users have been assigned different types of loads alongwith consumption limits and bounds. For this purpose, utility and end users communicate via AMI in order to schedule generation and consumption. In [21], game theoretic approach is used to optimize energy consumption patterns of appliances. Authors stated that there is a tradeoff between user comfort and electricity cost reduction. User comfort and cost reduction objectives are handled in a simple way such that users are given priority to choose either comfort or cost reduction.

In [22], energy management by controlling the thermostat of appliances is discussed. The thermostat of the appliance is controlled as per customer preferences to maintain the temperature in a desired limit. These types of approaches are efficient in terms of both cost and energy, however, it is difficult to tackle all the thermal constraints and parameters when different types of appliances are used. In [23], HVAC scheduling scheme is proposed. To maximize the user comfort, thermal constraints are considered in the optimization problem. For this purpose, thermodynamic model of a house is proposed to control the temperature. Moreover, this scheduling problem is solved by using nonlinear optimization techniques. In [24,25], user comfort is taken into consideration by controlling thermostats according to desired temperature set points. By making thermostats more intelligent, appliances can be controlled more efficiently, because appliances only operate when needed. Similarly, Blerim et al. [26] added intelligence to conventional programmable communication thermostats (CPCTs) by scheduling appliances via machine learning and wireless sensors. Along with electricity cost, energy consumption is also reduced as compared to other scheduling schemes where the primary objective was to reduce electricity cost only. Furthermore, user comfort is also maximized because once the pattern is learnt, energy management controller (EMC) takes actions according to energy demand and user preferences. In conclusion, some of the existing techniques are useful in reducing electricity cost while others are comfort aware. However, none of the techniques is efficient in scheduling appliances based on customers presence, weather conditions, and price signals. Therefore, to efficiently manage the energy consumption through appliance scheduling, all possible constraints must be taken into consideration.

In this paper, our main objective is to reduce the electricity cost of end users by scheduling home appliances while maintaining user comfort and PAR. Based on type, the household appliances are either controlled by intelligent thermostat or a controller which is run by GA. Various types of 
household appliances are considered where some are controlled by intelligent thermostats while other ones are controlled by using GA. For user comfort, appliance waiting is considered and iel appliances are used having fixed time delay set by users. Furthermore, user can set waiting time to minimum time slots according to his desire. On the other hand, knapsack capacity limit is imposed in optimization problem to reduce PAR. In each time slot, optimization algorithm schedules the appliances under capacity limit. Simulation results show that proposed algorithms achieve maximum electricity cost saving and average user comfort while minimizing the PAR.

\section{System Model}

We consider a residential unit where users share common energy source provided by utility. Each user has different types of $n$ appliances with variable energy consumption requirements. On the bases of user requirements, these appliances are categorized into $t c, u a, e l$, iel, and $r$. Within these categories, some appliances are shiftable having the objective of cost reduction. While, some appliances have strict schedules with aim at maximizing end user comfort. Figure 1 shows a schematic diagram of $t c$ and $u a$ appliances which are controlled by considering user occupancy and thermal constraints. The EMC uses the mathematical models of these appliances (Section 3.3), user preferences and external parameters (i.e., price signal, user presence and temperature set points) to generate the optimization schedules for a given time interval $t_{n}$. On the other hand, Figure 2 shows an architecture of $e l$, iel, and $r$ appliances. Here, the energy demand of $r$ appliances is fulfilled through electricity generated from local generator (microgrid, Section 3.1). This is because these are continuously running appliances which are difficult to schedule through optimization algorithms, hence do not take part in demand response (DR) programs. On the other hand, el and iel are scheduleable appliances and energy demand of these appliances is fulfilled through energy provided by utility company [25]. Moreover, the scheduling horizon and operating hours of all the appliances can vary and usually depend on the user preferences and appliance type. So, prior to the formulation of electricity cost reduction objective function, classification of appliances, user comfort and energy generation are discussed along with their constraints. More importantly, the proposed model is generic in nature and appliances considered in these categorization are not fixed. Any one who want to use this model can add or remove any appliances according to his own requirements.

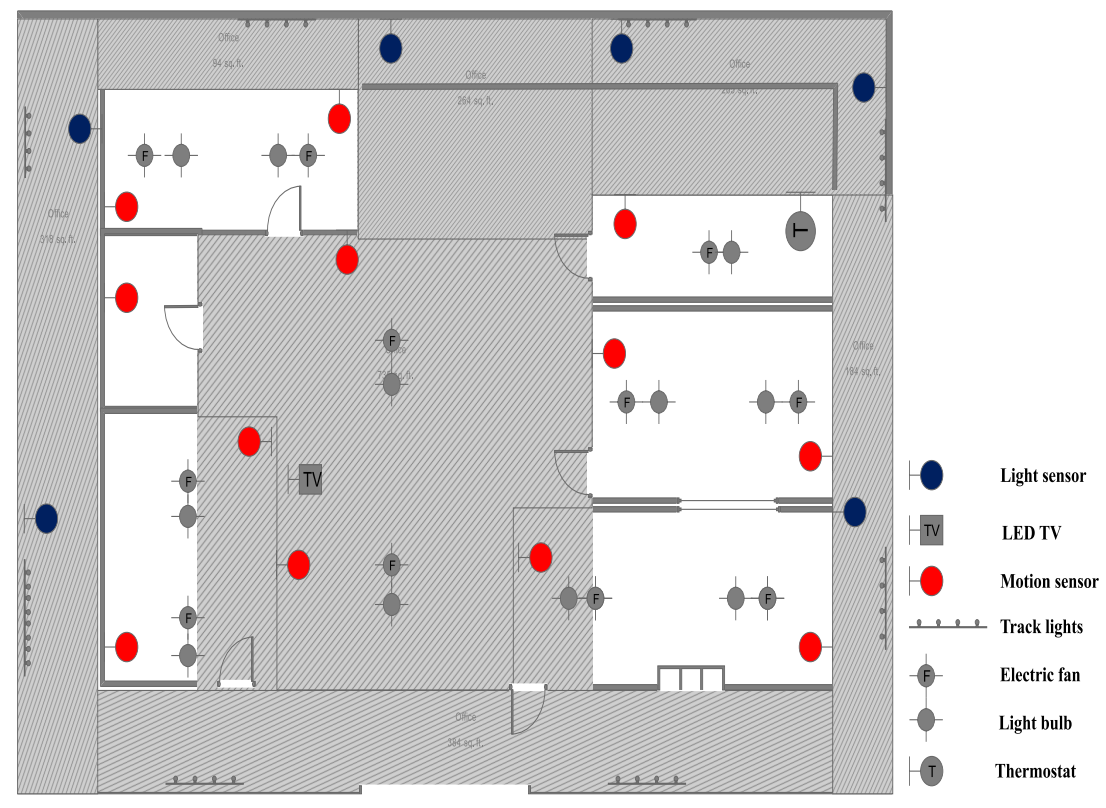

Figure 1. Conceptual diagram of $t c$ and $u a$ appliances. 


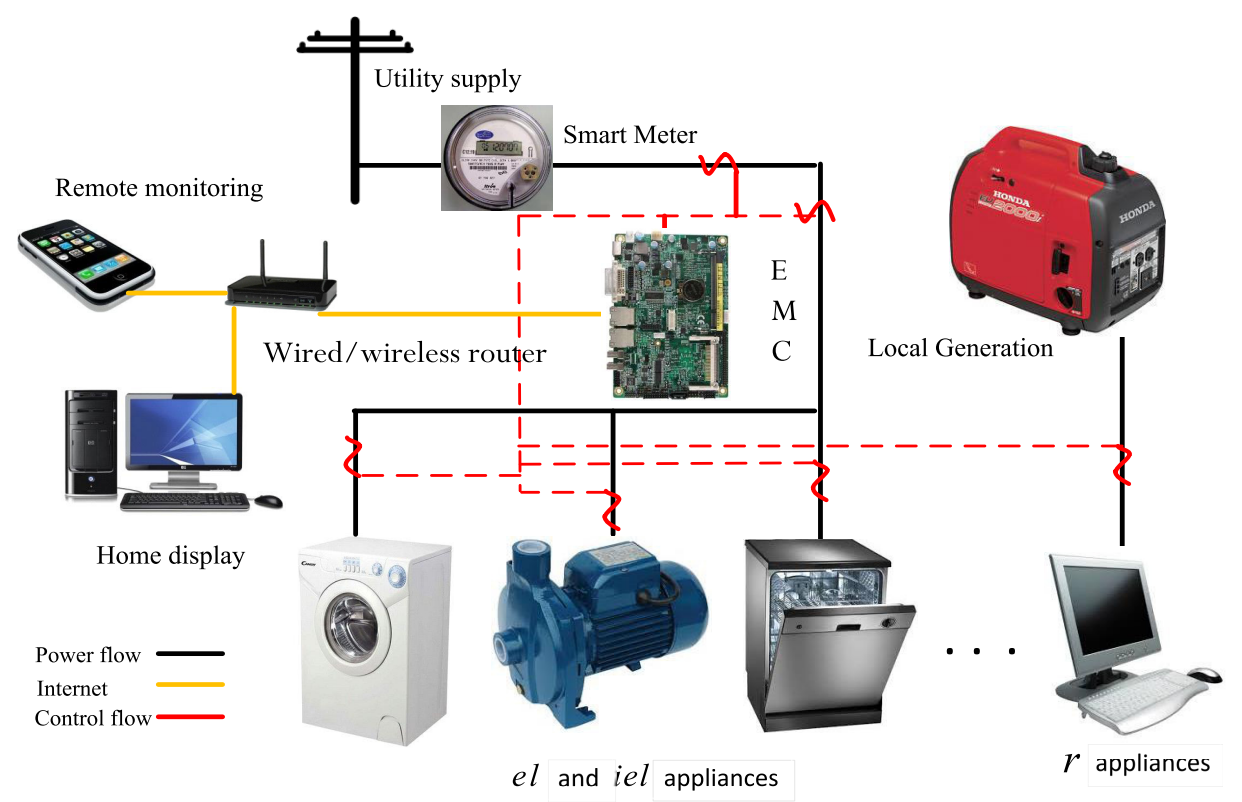

Figure 2. System model of $e l$, iel and $r$ appliances. EMC: energy management controller.

\subsection{Energy Generation Model}

A microgrid contains localized electricity sources i.e., diesel generators and renewable energy and loads. Loads normally connected with traditional grid (macrogrid). However, with the economic and physical conditions, it can be disconnected from macrogrid [27]. In the proposed work, we use localized diesel power generator operated at minimum fuel cost to meet the local energy consumption demand of $r$ appliances. Because, $r$ appliances are considered to be continuously running throughout the day and do not take part in DR program. The fuel cost minimization problem is formulated as [28]:

$$
O b j=\min \sum_{i=1}^{u} \iota_{i} P_{i}^{2}+\kappa_{i} P_{i}+c_{i}
$$

s.t:

$$
\begin{aligned}
& P_{i}^{\min } \leq P_{i} \leq P_{i}^{\max } \\
& \sum_{t=1}^{t n} \sum_{i=1}^{n} P_{i, t}=E_{r_{(i, t)}}
\end{aligned}
$$

where:

$-\iota_{i}$ : cost due to generator losses

$-P_{i}^{2}$ : power produced by generator in micrgrid

$-\kappa_{i}$ : power generation coefficient of a generator

$-c$ : cost dur to generator startup fuel consumption

$-P_{i}^{\min }:$ minimum power produced by a generator

$-P_{i}^{\max }$ : maximum power produced by a generator

$-E^{r}$ : total energy demand of $r$ appliances

Equation (1a) shows that total generated power must be within the maximum and minimum capacity. While Equation (1b) shows that total generated power must be equal to power required to run $r$ appliances. The above optimization problem is solved by using GA.

The main steps to solve economic load dispatch problem through GA are discussed in (Section 4, Figure 3). As the algorithm processes, new population is produced by linear crossover and real coded mutation. Whereas, rollet wheel selection is used for reproduction of the new population. 


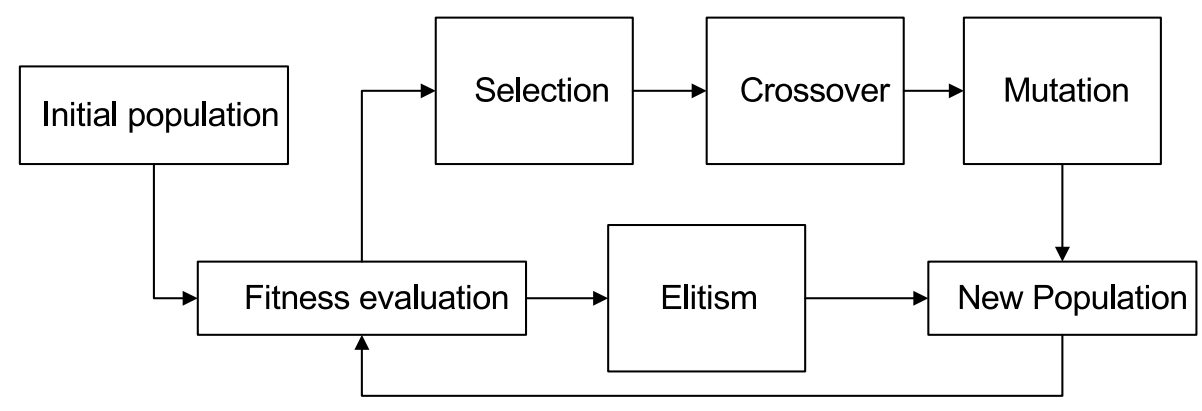

Figure 3. Main steps of genetic algorithm (GA).

\subsection{User Frustration}

In load scheduling problems, $\mathrm{el}$ and iel appliances (Sections 3.3.3 and 3.3.4) are shifted from on-peak hours to off-peak hours while aiming electricity cost reduction (Figure 4). In this case, users may face surplus delay in terms of appliance waiting time with high probability of exceeding a bearable limit which ultimately affects user comfort. To avoid user frustration, our objective here is to reduce appliance waiting time along with electricity cost reduction of the $e l$ and iel appliance. For this purpose, we formulate user frustration as a function of appliance average waiting time which is calculated as [21]:

$$
D_{i}^{a}=\frac{\sum_{t=1}^{t n} \sum_{i=1}^{u}\left|\left(t_{i, t}^{\mathrm{u}-\mathrm{sch}}-t_{i, t}^{\mathrm{sch}}\right)\right|}{t_{l_{i}}} \quad \forall t n
$$

where, $D_{i}$ is appliance delay due to appliance shifting which is difference between appliance $t^{\mathrm{u} \text {-sch }}$ and $t^{\text {sch }}$ time. The scheduling algorithm adjusts the appliances before or after specified time in response to electricity price and user preferences. The appliance waiting time can be negative (left hand side of Figure 4) if appliance is scheduled before specified time and vice versa. The maximum waiting time that an appliance can bear is calculated as follows:

$$
D_{i}^{m}=t n-t_{l i}
$$

where, $D_{i}^{m}$ is the maximum permissible delay of any appliance. User frustration increases if we increase $D_{i}^{a}$. In worst case, the user frustration is maximum, when $D_{i}^{a}$ is equal to $D_{i}^{m}$. The user frustration $\lambda_{i}$ is calculated as follows:

$$
\lambda_{i}=\frac{D_{i}^{a}}{D_{i}^{m}}
$$

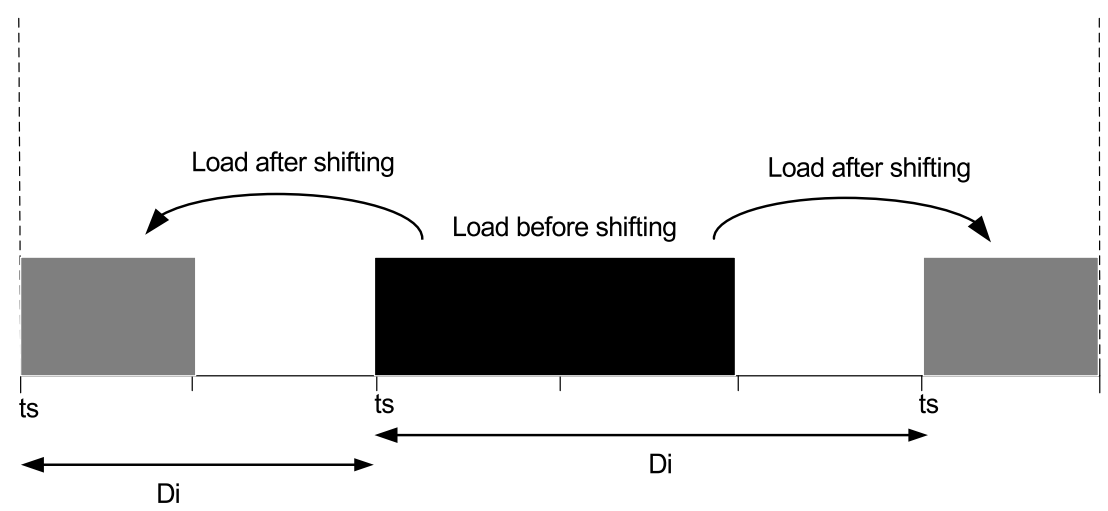

Figure 4. Appliance waiting time. 


\subsection{Types of Appliances}

Within the house, each appliance consumes variable amount of energy based on energy demand and customer preferences. So, we group these appliances based on user comfort maximization and electricity cost reduction perspectives. These categorizations are only used to analyze the energy consumption behaviours based on associated constraints and its impact on end user comfort. We have selected non overlapping appliances (having unique set of constraints) in the refined version of manuscript. Thus, the on/off schedules are no longer ambiguous.

\subsection{1. tc Appliances}

Typically, the working of $t c$ appliances is controlled by CPCT. In this paper, the appliances are controlled not only by considering thermal constraints but also human occupancy. Along with other operational constraints, the working of these appliances also depends on thermal constraint such as temperature. HVAC and refrigerator are examples of appliances that belong to tc category and appliances other than these two which meet the given criteria can also be included in this category. The details of mathematical models and working of tc controlled appliances are given as follows:

HVAC

Energy consumption of HVAC depends upon two factors: (i) total number of occupants in the room and; (ii) temperature. Here, we aim to minimize electricity cost of the consumers while taking into consideration their comfort level. In most of the traditional energy management techniques, appliances are scheduled based on low electricity cost which overshadow comfort. To maintain room temperature within specified limits [tem $\left.e_{r}^{\min }, t e_{r}^{\max }\right]$, HVAC consumes total energy $E^{h}$ as follows [29]:

$$
\begin{gathered}
E^{h}=\sum_{t=1}^{t n} e_{t}^{h} \cdot \varphi_{t} \\
t e_{r}^{\min } \leq t e_{r} \leq t e_{r}^{\max } \\
e_{\circ}^{h} \leq e^{h} \leq e_{\bullet}^{h} \\
e_{\bullet}^{h}=e_{\circ}^{h}+\alpha \beta e^{h}
\end{gathered}
$$

The occupancy value is calculated as:

$$
\alpha= \begin{cases}1 & \text { if person is present at time } t \\ 0 & \text { else }\end{cases}
$$

If the temperature is within given limits, the value of $\beta$ variable is 0 . Otherwise, its value is equal to 1 . The $\beta$ variable is used for simulation purpose which is denoted as follows:

$$
\beta= \begin{cases}1 & \text { if temperature is not within limits } \\ 0 & \text { else }\end{cases}
$$

where:

$-E^{h}$ : total energy consumed by HVAC

$-t n$ : total number of time slots which are 24

$-t e_{r}^{\min }:$ minimum temperature of a room

$-t e_{r}^{\max }$ : maximum temperature of a room

$-e_{\circ}^{h}$ : energy consumed by HVAC when there is no occupant in the room

$-e^{h}$ : energy consumed by HVAC when there are occupants in the room

$-\alpha$ : shows the state of person presence in the room $[0,1]$

$-\varphi$ : appliance on/off status 
Equation (5a) represents that room temperature varies within specified limits, $\left(20-26{ }^{\circ} \mathrm{C}\right)$. As temperature deviates from given set points, HVAC needs more energy to keep temperature within given limits and vice versa. So, the energy consumption range in the presence and absence of occupant(s) is represented by Equation (5b). Energy consumption of HVAC is varied with the number of occupants in the room. Equation (5c) shows the presence of occupant(s), such that energy consumption of HVAC is $\alpha$ times increased. In conclusion, these constraints show that temperature has direct impact on the energy consumption of HVAC.

\section{Refrigerator}

We aim to minimize the daily monitory expense related to the power consumption of the refrigerator while considering thermal constraints of the refrigerator. Inside temperature of the refrigerator must be within temperature range of $\left[t e_{\min }^{\text {ref }}, t e_{\max }^{\text {ref }}\right]$. To keep refrigerator's inside temperature within acceptable limits, total number of door open attempts and duration must be at minimum because these attempts and the associated duration directly affect energy consumption. The energy consumption of refrigerator is given as [29]:

$$
\begin{gathered}
E^{\mathrm{ref}}=\sum_{t=1}^{t n} e_{t}^{\mathrm{ref}} \varphi_{t} \\
t e_{\min }^{\mathrm{ref}} \leq t e_{n}^{\mathrm{ref}} \leq t e_{\max }^{\mathrm{ref}}
\end{gathered}
$$

where:

$-E^{\text {ref: }}$ total energy consumed by refrigerator

$-t e_{\max }^{\text {ref }}$ : maximum temperature limit of a refrigerator

$-t e_{\mathrm{min}}^{\mathrm{ref}}:$ minimum temperature limit of a refrigerator

Here the $E^{\text {ref }}$ is equal to the sum of energy consumption in standby $e_{\mathrm{S}}$, compressor $e_{\mathrm{c}}$, with door open attempts $e_{\mathrm{i}_{\mathrm{do}}}$ and with temperature $e_{\mathrm{t}_{\mathrm{in}}}$, respectively. Refrigerator consumes $\left(\beta \times e_{\mathrm{i}_{\mathrm{do}}}\right)$ times more energy on each door open attempt. Similarly, refrigerator consumes $\left(\gamma \times e_{\text {tin }}\right)$ times more energy with outside temperature variations. It means, energy consumption of a refrigerator depends upon its temperature variations. Equation (6a) represents the internal temperature bounds of a refrigerator.

\subsection{2. ua Appliances}

These types of appliances are turned on or off on the basis of human occupancy. For this purpose, we assume that different sensors are deployed in the room in order to detect human presence (Section 5). When any sensor detects human presence, their respective appliance(s) are turned on and energy consumption is calculated. By using human presence mechanism, unnecessary appliances can be turned off to reduce uneven energy consumption. Moreover, any appliance which meets the $u a$ criteria, can be considered in this category. Total energy consumption of $u a$ appliances can be calculated as given in [29]:

$$
E^{u a}=\sum_{t=1}^{t n} \sum_{i=1}^{n} e_{i, t}^{u a} \varphi_{i, t}
$$

where:

$$
E^{u a}=\sum_{\delta=1}^{\delta_{n}} e_{\delta}^{u a}, \forall u a
$$

where:

$-e^{u a}:$ energy consumption of a $u a$ appliances

$-\delta_{n}$ : the $n$ occupancy sensors in the room 
Equation (7a) shows that total energy consumption of $u a$ appliances depends upon $\delta$ parameter. The appliances associated with these sensors remain on or off based on the values of these sensors.

\subsection{3. el Appliances}

These are fixed energy consumption appliances, however, these are interruptible whose working can be scheduled throughout the time interval. In order to save electricity cost, these appliances are scheduled in low pricing hours. Total energy consumption of these types of appliances is calculated by using the following equation:

$$
E^{e l}=\sum_{t=1}^{t n} \sum_{i=1}^{n} e_{i, t}^{e l} \varphi_{i, t}
$$

$e l$ appliances perform their tasks under the following constraints:

$$
\begin{aligned}
& \varphi_{i, t}=t_{l_{i}}, \forall n \\
& \sum_{t=1}^{t n} \sum_{i=1}^{n} e_{i, t} \cdot \varphi_{i, t} \leq \vartheta_{t}, \forall e l \\
& \tau_{i} \leq t n-t_{l_{i}} \quad \forall e l \\
& D_{i}=\left|\left(t_{i}^{\mathrm{u}-s c h} \times \varphi_{i, t}\right)-\left(t_{i}^{\mathrm{sch}} \times \varphi_{i, t}\right)\right| \forall \varphi_{i, t} \neq 0\left|D_{i} \leq 3\right| t \in t n \\
& \varphi_{i, t} \in\{0,1\}
\end{aligned}
$$

where:

$-t_{l_{i}}:$ appliance duty cycles (total operating hours)

$-\vartheta$ : energy consumption capacity limit used for PAR reduction

$-\tau$ : total time in which appliance can be scheduled (appliance scheduling horizon)

$-D_{i}$ : appliance total waiting time occurs due to rescheduling

$-t_{i}^{\mathrm{u}-\mathrm{sch}}$ : appliance unscheduled starting time initially set by users

$-t_{i}^{\text {sch }}$ : appliance scheduled starting time set by energy scheduler

Equation (8a) represents that unscheduled and scheduled $t_{l_{i}}$ are equal. Total energy consumption of $e l$ appliances is within energy constraints $\vartheta_{t}$ which is given in Equation (8b). Whereas, Equation (8c) represents the scheduling horizon of $e l$ appliances. As these types of appliances are interruptible so their operating time may vary in scheduling horizon. Equations $(8 c, 8 d)$ represent the appliance waiting time and on/off status, respectively.

\subsection{4. iel Appliances}

These types of appliances are interruptible and continue their operation once turned on. User can not compromise on comfort, so these appliances are turned on when electricity price is low and complete their duty cycle without interruption. Energy consumption of these types of appliances is calculated by using the following equation:

$$
E^{i e l}=\sum_{t=1}^{t n} \sum_{i=1}^{n} e_{i, t}^{i e l} \varphi_{i, t}
$$


The associated constraints for iel appliances are listed as follows:

$$
\begin{aligned}
& \varphi_{i, t}=t_{l_{i}}, \forall n \\
& \sum_{i=1}^{n} \sum_{t=1}^{\text {tn }} e_{i, t} \cdot \varphi_{i, t} \leq \vartheta_{t}, \forall e l \\
& t_{i}^{\text {sch }} \leq \tau_{i} \leq t_{i}^{\text {sch }}+t_{l_{i}} \quad \forall i e l \\
& D_{i}=\left|\left(t_{i}^{\mathrm{u}-\mathrm{sch}} \times \varphi_{i, t}\right)-\left(t_{i}^{\mathrm{sch}} \times \varphi_{i, t}\right)\right| \forall \varphi_{i, t} \neq 0\left|D_{i} \leq 3\right| t \in t n \\
& \varphi_{i, t} \in\{0,1\}
\end{aligned}
$$

Equation (9a) represents that appliance unscheduled $t_{l_{i}}$ must be equal to scheduled $t_{l_{i}}$. Equation (9b) shows that total energy consumption of iel appliances must not exceed $\vartheta_{t}$. Because these appliances are uninterruptible, so they have continuous $t_{l_{i}}$ after scheduling. Total scheduling horizon of these appliances is given in Equation (9c). Waiting time of these appliances is not more than $3 \mathrm{~h}$ as we impose this limit. Equation (9d) represents the total waiting time. Appliances on/off status is represented by Equation (9e).

\subsection{5. $r$ Appliances}

We consider that these appliances are continuously running during whole day. So, these appliances do not take part in DR programs and thus can not be scheduled. Energy consumption of $r$ appliances is calculated by using the following equation:

$$
E^{r}=\sum_{t=1}^{t n} \sum_{i=1}^{n} e_{i, t}^{r}-E_{t}^{\mathrm{g}}=0
$$

where, $E_{t}^{g}$ is total energy generated by local generator, $e_{i, t}^{r}$ is energy consumption of $r$ appliances and $E^{r}$ is total energy consumption of $r$ appliances, respectively.

$$
\tau_{i}=t_{i}^{s} \operatorname{ch} \quad \forall r, t_{i}^{\mathrm{sch}} \in t n
$$

\subsubsection{Total Energy Consumption}

The total energy consumption $E_{\mathrm{T}}$ of all types of appliances including $e l, i e l, r, u a$, and $t c$, is calculated as follows:

$$
E_{\mathrm{T}}=\sum_{t=1}^{t n} \sum_{i=1}^{n}\left(E_{i, t}^{e l}+E_{i, t}^{i e l}+E_{i, t}^{r}+E_{i, t}^{u a}+E_{i, t}^{t c}\right)
$$

The daily total electricity $\operatorname{cost} C_{\mathrm{T}}$ of these appliances is given as:

$$
C_{\mathrm{T}}=\sum_{t=1}^{t n}\left(E_{\mathrm{T}_{t}} \varsigma_{t}-E_{t}^{r} \varsigma_{t}\right)
$$

Equation (13) shows that $C_{\mathrm{T}}$ is equal to the electricity taken from grid. The second part of Equation (13) $E_{t}^{r}$ denotes the energy demand of $r$ appliances is fulfilled through microgrid model (Section 3.1). The final cost minimization objective function is given as:

$$
O b j=\min \sum_{t=1}^{t n} \sum_{i=1}^{n} C_{\mathrm{T}}
$$




\subsection{Peak to Average Ratio Reduction}

Along with electricity price, PAR reduction of the system is also taken into consideration to increase the stability of the grid. For this purpose, we introduce energy consumption capacity limit in the optimization problem. PAR can be calculated by using the following expressions:

$$
\begin{array}{cc}
p_{\mathrm{m}}=\max \left(\sum_{t=1}^{t n} \sum_{i=1}^{n} E_{i, t}^{e l}+E_{i, t}^{i e l}\right) & \forall[t \in t n] \\
p_{\mathrm{a}}=\frac{\left(\sum_{t=1}^{t n} \sum_{i=1}^{n} E_{i, t}^{e l}(t)+E_{i, t}^{i e l}\right)}{t n} & \forall[t \in t n] \\
P A R=\frac{p_{\mathrm{m}}}{p_{\mathrm{a}}} &
\end{array}
$$

where $p_{\mathrm{m}}$ is maximum peak and $p_{\mathrm{a}}$ is average peaks.

By reducing PAR, we can achieve system stability and increase spinning reserves capacity of the power system. Moreover, reduction in peak load demand leads to reduction in peak power plant cost and transmission losses.

Note: When a person is in a room, he/she would like that the temperature is already within the limits, or close to them: if the HVAC is off whenever nobody is in the room, the temperature might get very far from the limits, causing an unpleasant environment most of the times someone gets in the room. However, for this purpose, we need to predict human behaviors based upon their daily, weekly, or monthly schedules. Then based on these behaviors, we can predict when a person will probably enter the room/house and schedule HVAC, accordingly. This might increase total energy consumption as behaviors and schedules are dynamic but at the same time increasing end user comfort in terms of balanced room temperature. In conclusion, advanced/intelligent prediction techniques are needed, which demand research work beyond the scope of this paper. For the sake of simplicity in implementation, this research work carries out implementation with the assumption of uniformly distributed person arrival to the room.

\section{Genetic Algorithm Based Energy Management Algorithms}

Typical scheduling optimization problems have discrete, non-linear and highly constrained search space which make desired solutions very difficult to find. Various mathematical optimization techniques are being used to solve these types of problems and it is impossible that single algorithm gives best optimal solution to different optimization problem. Among these, EAs are extensively used for solving such types of complex optimization problems due to fast convergence and ability to deal with different dynamics of the problems. Furthermore, these algorithms are efficient in providing optimal solutions when other mathematical optimization algorithms fail to provide [30]. Therefore, we use GA to solve the complex energy optimization and appliance scheduling problem.

There are seven major steps of GA: initialization of parameters, fitness evaluation, selection, crossover, mutation, elitism and generation of new population. The details of these steps are given as follows:

Initial Population: In first step, random population which consists of candidate solution (chromosomes) is initialized. The initial population is in $n x m$ matrix form, where $m$ and $n$ represent population size and total number of appliances, respectively. The matrix $A_{m \times n}$ is written as follows: 


$$
A=\left[\begin{array}{ccccc}
A_{11} & A_{12} & A_{13} & \ldots & A_{1 m} \\
A_{21} & A_{22} & A_{23} & \ldots & A_{2 m} \\
A_{31} & A_{32} & A_{33} & \ldots & A_{3 m} \\
\vdots & \vdots & \vdots & \ddots & \vdots \\
A_{n 1} & A_{n 2} & A_{n 3} & \ldots & A_{n m}
\end{array}\right]
$$

Fitness Evaluation: In this step, fitness of randomly initialized population is evaluated such that if chromosome ( $\mathrm{m}=$ total no. of appliances) is $\left[\begin{array}{llllll}1 & 0 & 1 & 1 & 1 & 0\end{array}\right]$, it means appliances $1,3-5$ are on. We then calculate cumulative power and multiply it with electricity price to calculate energy consumption cost. This process continues until the evaluation of population's fitness. This process gives an optimal pattern of household appliances at time slot $t$ whose electricity cost is low.

Selection: At the start of the algorithm, random population is initialized only once. In the next generation, new population is generated through mutation and crossover operators. For this purpose, a random pair form random population is selected to form new population. We use tournament based selection procedure to select a random pair.

Cross over and Mutation: After the selection of random pair, new population of chromosomes is produced by using mutation and crossover operators. There have been different methods of crossover and mutation, and we use binary mutation and single point crossover methods. Convergence of GA depends on mutation and crossover rate. Higher crossover means faster convergence. On the other hand, high mutation rate may diverge optimal solution, which means slow convergence.

Elitism: Sometimes, near optimal solution is found at the start of algorithm but after mutation and crossover, it may be lost. So, elitism is used to save this solution for evaluation purpose. As we discussed earlier that GA has comparatively faster convergence rate where the convergence mainly depends upon crossover and mutation probability. Higher crossover probability results faster convergence and higher mutation probability results less optimal solution and vice versa. Moreover, improper adjustment of these parameters may result premature convergence of the algorithm. However, in some cases the best optimal solution can be found earlier and after that crossover and mutation may variate this solution. So, elitism mechanism is used to keep the optimal solution in the next step from one generation to another to get final optimal solution. The parameters based on which the optimal schedules of different appliances are generated are shown in Table 2. Based on RTP signal, energy demand and user requirements, GA generates the optimal patterns of all appliances in each time slot. For example, if we have six appliances, six bit binary pattern (011001) is generated where 0 shows off and 1 shows on state of appliances. In each time slot, this process repeats to obtain optimal patterns because electricity prices vary based on energy demand of the appliances and user preferences.

Table 2. Control Parameters of GA.

\begin{tabular}{ccc}
\hline Parameter & Symbol & Value \\
\hline Chromosome length & $\mathrm{N}$ & 4 \\
Maximum generation & $g_{\max }$ & 500 \\
Population size & $p_{\text {size }}$ & 200 \\
Crossover rate & $\mathrm{Pc}$ & 0.9 \\
Mutation rate & $\mathrm{Pm}$ & 0.1 \\
\hline
\end{tabular}

\subsection{Complexity of Genetic Algorithm}

In GA implementation, representation of solution is vital to its convergence and processing time (speed). GA algorithm produces the best solution by evaluating the initial random population. The size of initial population usually depends on the nature of the problem. The algorithm takes more processing time to evaluate the fitness of all individuals for large population size and vice versa. 
Figure 5 shows the analysis of processing time and fitness function w.r.t population size and total number of appliances. It is clear from Figure $5 \mathrm{a}$ that processing time increases by increasing total number of appliances in time $t$. It is also shown that algorithm takes more processing when initial population size is large as compared to small population size. This is because the algorithm evaluates $A_{m \times n}$ matrix which can take more processing time while taking into consideration all constraints (Equations (9a-9e)). In Figure 6, processing time of GA in terms of total number of time slots has been shown. It is clear from the figure that processing time (convergence time) increases as we increase the total number of time slots during time $t$ and vice versa. This is due to the fact that algorithm needs more time to find optimal time slots when total number of time slots are increased. In the proposed work, we use 24 and 48 time slots for simulation purpose to evaluate the performance of GA in terms of convergence, processing time and optimal solution Figures 5 and 6 . The proposed algorithm has the capability to schedule home appliances considering any number of time slots, but at the cost of more processing time. In conclusion, complexity of the proposed algorithm increases as we increase the total number of appliances, time slots and initial population size.

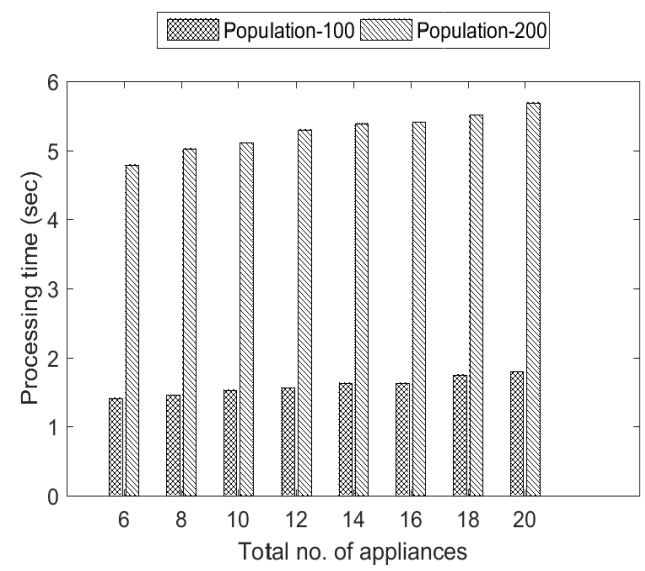

(a)

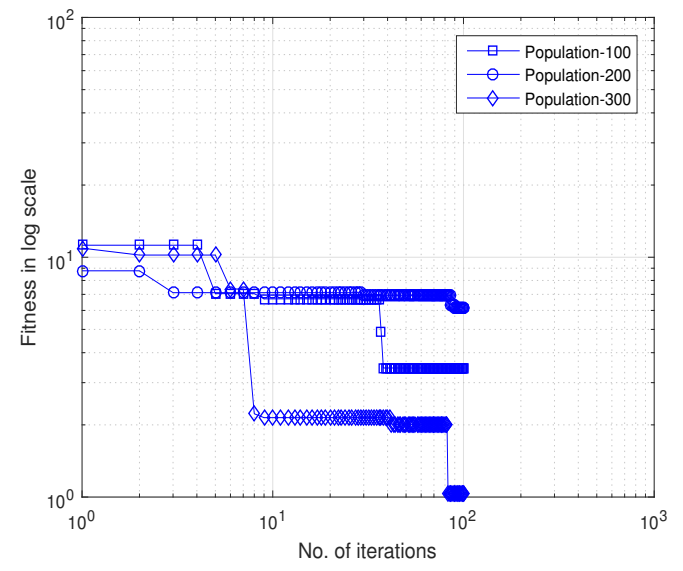

(b)

Figure 5. Comparison of processing time and fitness of GA w.r.t; (i) total no. of appliances; (ii) population size: (a) processing time of GA; and (b) fitness of GA.

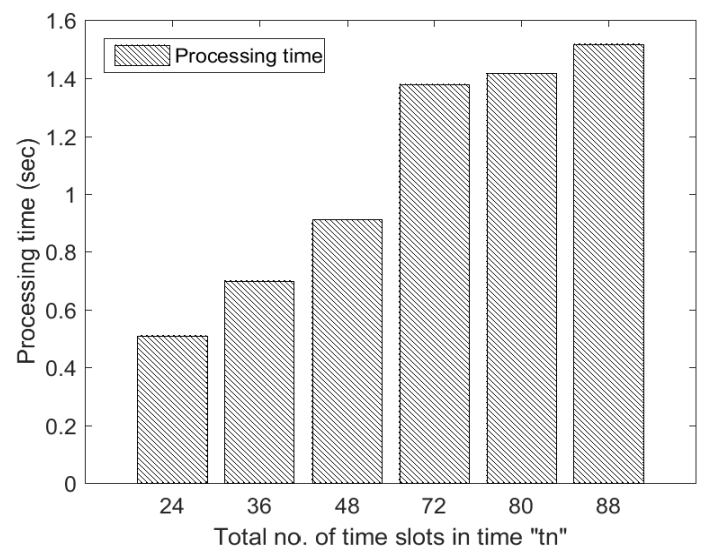

Figure 6. Analysis of processing time of GA w.r.t total number of time slots (for single appliance only). 


\section{Simulations and Results}

In this section, we discuss the simulation results and assess the performance of the proposed energy management algorithms in terms of energy consumption, electricity cost savings and thermal comfort of the consumer. Different types of appliances, i.e., $t c, u a, e l, i e l, r$, having variable energy demand and operating time have been considered in this work. The working of tc appliances is controlled by taking into consideration human occupancies and thermal constraints. The $u a$ appliances are turned on only when any person is present in the room. For this purpose, we assumed different sensors placed in the room which detect human motion/presence and provide signal to EMC [31]. Based on this information, EMC decides to turn that particular appliance on or off. The $e l$ appliances are those which can be scheduled throughout the day. Moreover, these appliances are interruptible in nature and EMC schedules these appliances for cost reduction perspective without violating $l_{t_{i}}$. On the other hand, the iel appliances are scheduleable but non-interruptible in nature. Once these appliances are turned on, they will complete their working hours. At the end, the energy demand of $r$ appliances is fulfilled through local generation because these are continuously running appliances as discussed in Section 3. The details about the appliances used in these categories and simulation parameters are given in Tables 1-6, respectively. The RTP signal is directly obtained via smart meter which later on is utilized by EMC for appliance scheduling and controlling (Figure 7, [32]).

Table 3. Appliances energy consumption and operating time [33].

\begin{tabular}{cccc}
\hline Type of Appliance & Appliance & $\boldsymbol{t}_{\boldsymbol{l}_{\boldsymbol{i}}} \mathbf{( h )}$ & Power Rating (kWh) \\
\hline$t c$ & Refrigerator, HVAC & - & $1.24,1.10$ \\
$u a$ & Fans, Lights & - & $0.10,0.47$ \\
$e l$ & Washing machine, Dishwasher & 5,7 & $3.4,1.50$ \\
$i e l$ & Water pump, Oven & 8 & $3.5,1.5$ \\
$r$ & Computer, TV & 8 & $0.1,0.3$ \\
\hline
\end{tabular}

Table 4. HVAC parameters [34].

\begin{tabular}{cccccc}
\hline & 1 & 2 & 3 & 4 & 5 \\
\hline Appliance & Temperature range $\left({ }^{\circ} \mathrm{C}\right)$ & Desired temp. $\left({ }^{\circ} \mathrm{C}\right)$ & $E_{*}(\mathrm{kWh})$ & $E^{\mathrm{HVAC}}(\mathrm{kWh})$ & $\alpha$ \\
\hline HVAC & $20-26$ & 23 & 0.0175 & 3.5 & $0-4$ \\
\hline
\end{tabular}

Table 5. Refrigerator parameters [33,35].

\begin{tabular}{ccccccc}
\hline & 1 & 2 & 3 & 4 & 5 & 6 \\
\hline Appliance & Temperature Range $\left({ }^{\circ} \mathrm{C}\right)$ & $e_{\mathrm{s}}(\mathrm{kWh})$ & $e_{\mathrm{c}}(\mathrm{kWh})$ & $e_{\mathrm{i}_{\mathrm{do}}}(\mathrm{kWh})$ & $e_{\mathrm{t}_{\mathrm{in}}}(\mathrm{kWh})$ & $\beta, \gamma$ \\
\hline Refrigerator & $1-5$ & 0.5 & 2 & 0.0124 & 0.141 & $0-4,0-5$ \\
\hline
\end{tabular}

Table 6. Parameters of $u a$ appliances [34].

\begin{tabular}{cccc}
\hline & 1 & 2 & 3 \\
\hline Appliance Type & $e_{\mathrm{f}}(\mathrm{W})$ & $e_{1}(\mathrm{~W})$ & $\delta_{1}, \delta_{\mathrm{r}}, \Phi$ \\
\hline$u a$ & 100 & 28 & {$[0,1]$} \\
\hline
\end{tabular}




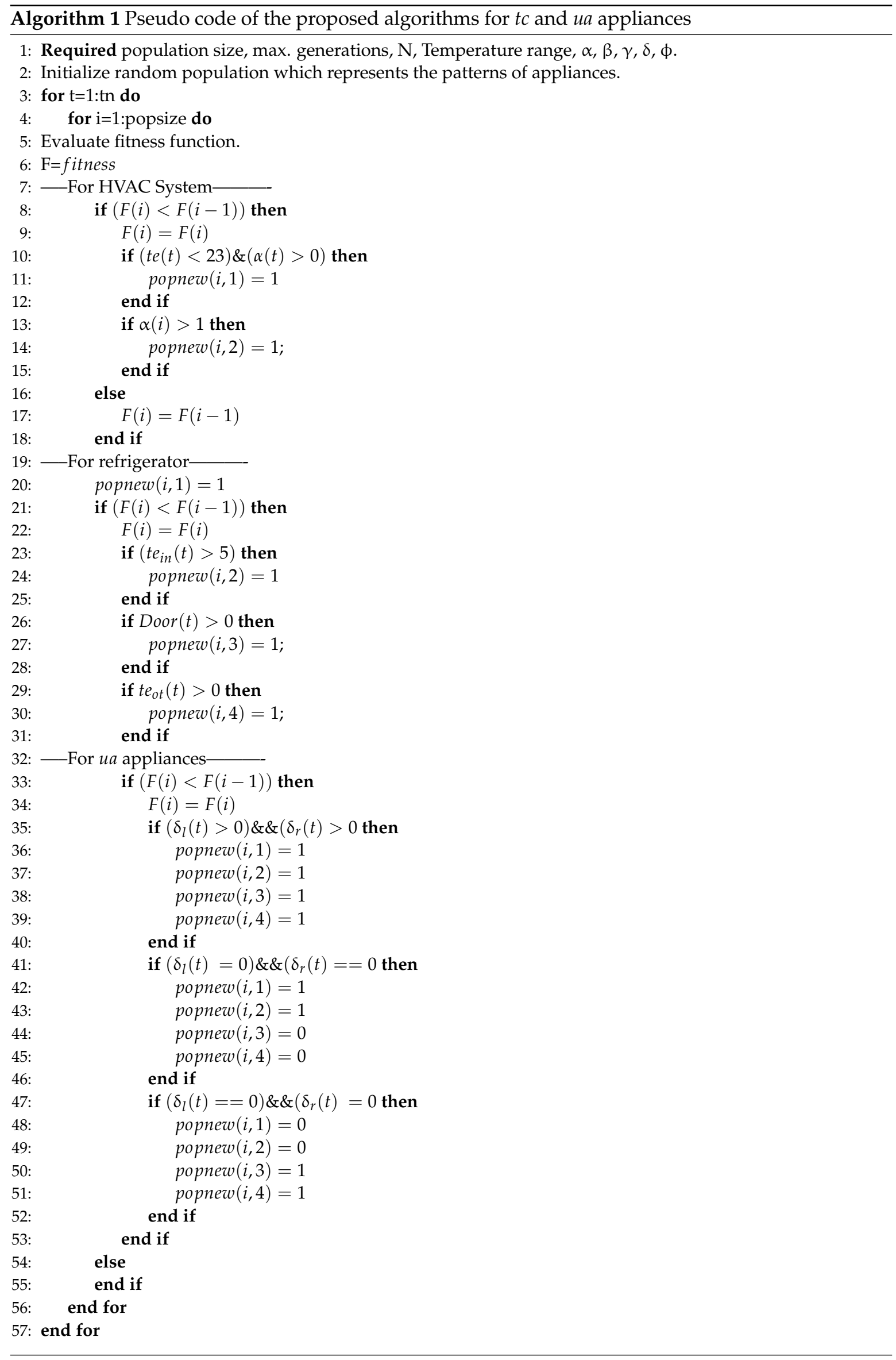




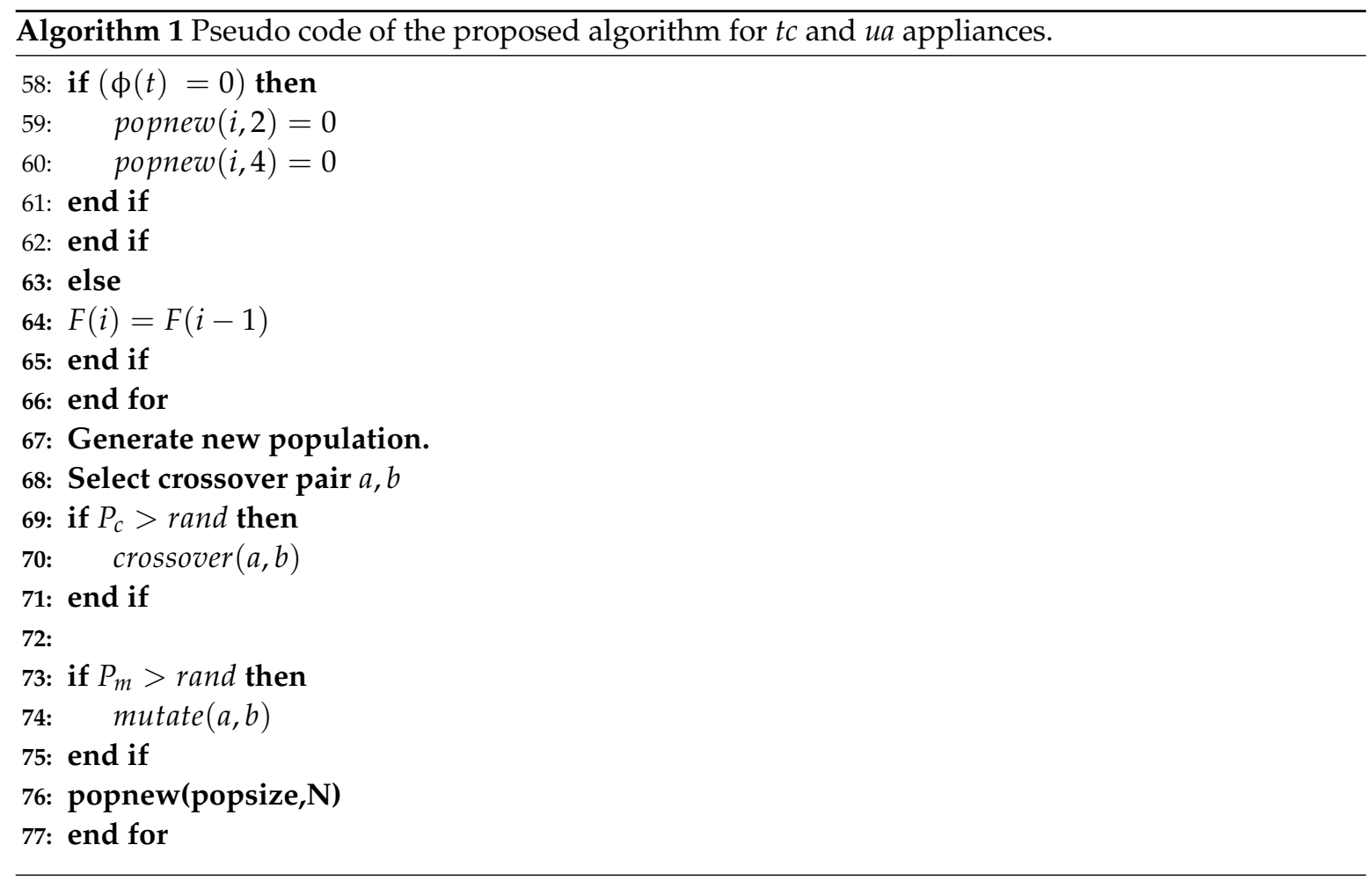

Figure 8 illustrates the energy consumption of HVAC in case of CPCT and IPCT. Using CPCT, HVAC turns on when indoor temperature falls below the threshold temperature $\left(t_{0} \rightarrow t_{14}\right)$, $\left(t_{15} \rightarrow t_{24}\right)$ and turns off when temperature level is maintained in the room $\left(t_{14} \rightarrow t_{15}\right)$. Turning on HVAC without taking into consideration the human occupancy may increase the energy consumption leading to high electricity cost. To enhance the appliance utility, the proposed algorithm considers all these factors (temperature and occupancy) and maintains the desired room temperature. It is clear from Figure 8 that when there is no occupant in the room $\left(t_{1} \rightarrow t_{2}\right)$ and $\left(t_{3} \rightarrow t_{4}\right)$ (red dotted line), EMC sends turn off signal to the iPCT. For simplicity, only occupants presence has been shown in Figure 8a based on which HVAC turns on or off. From $\left(t_{5} \rightarrow t_{7}\right)$ and $\left(t_{15} \rightarrow t_{19}\right)$, there is again no occupant in the room and iPCT turns off the HVAC according to (Section 3.3.1). In case of CPCT, HVAC is turned on during time slots $\left(t_{5} \rightarrow t_{7}\right)$ and $\left(t_{8} \rightarrow t_{9}\right)$ on the basis of temperature difference only (Figure 8). Although, there is no occupant found in these time slots which is an important reason of uneven energy wastage. During time slot $\left(t_{13} \rightarrow t_{14}\right)$, both CPCT and iPCT turn off HVAC because there is no occupant in the room and temperature is within desired limit. From above results, it is clear that iPCT is more efficient in handling appliance working as compared to CPCT. Figure 9 shows the resultant electricity cost of HVAC.

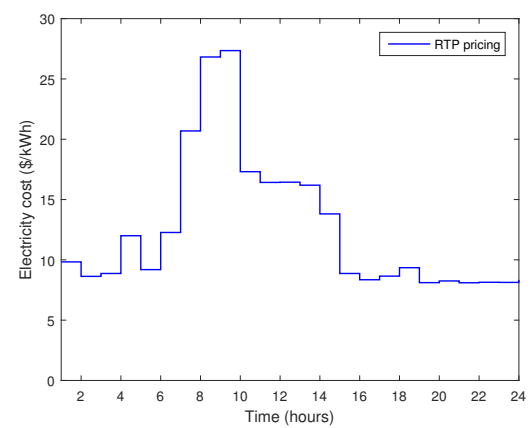

Figure 7. Real time price (RTP) pricing signal obtained from [32]. 

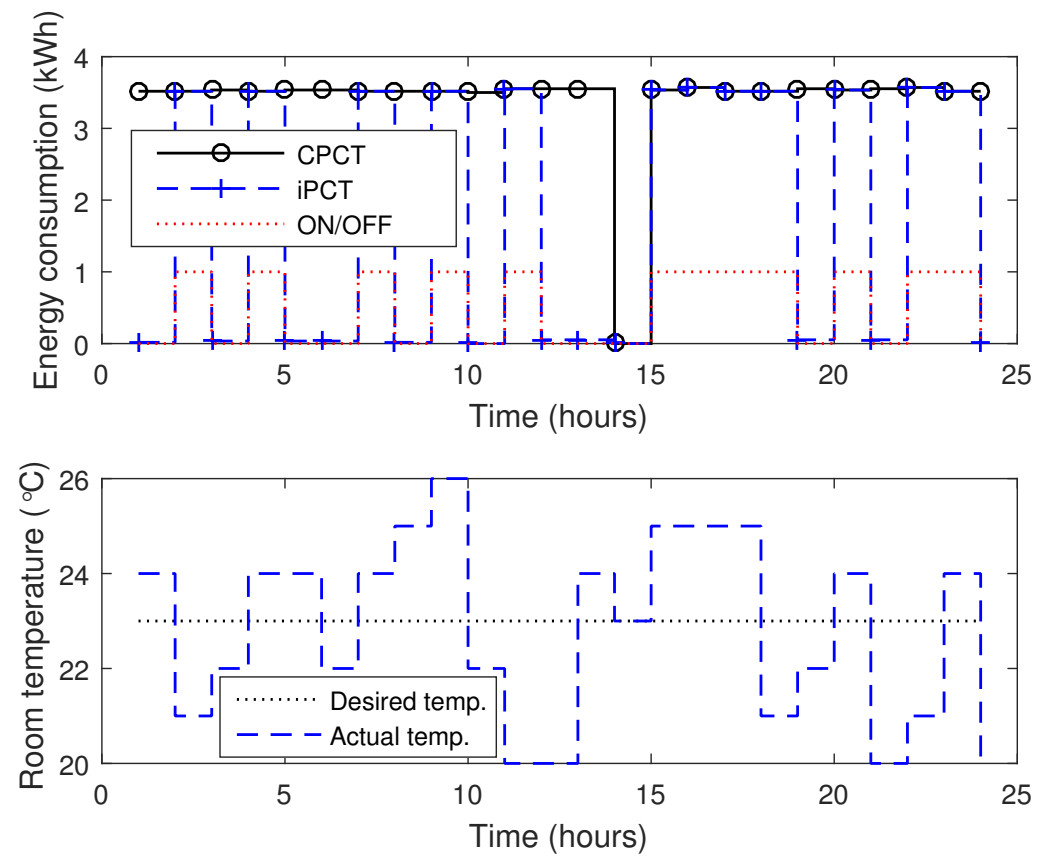

Figure 8. Energy consumption of HVAC w.r.t variation in room temperature with number of occupants in the room, (on-if person is present in the room, off-if person is not present in the room).

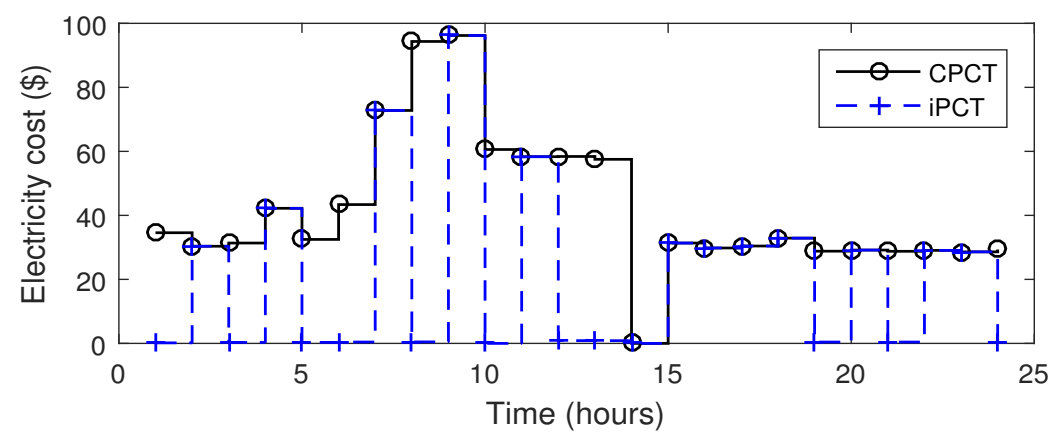

Figure 9. Energy consumption cost of HVAC.

In case of refrigerator, energy consumption depends on outside temperature variations and number of times the door is opened. The number of times the door opens in a single time slot is considered uniformly distributed random variable within the range of [0,4]. Each time when door is opened at $90^{\circ}$ for $12 \mathrm{~s}$, additional energy of $12.4 \mathrm{~W} / \mathrm{h} /$ day is consumed. It is clear that the door opening rate is directly proportional to the total duration required for user satisfaction. The iPCT turns on the refrigerator compressor only when the inside temperature exceeds $5^{\circ} \mathrm{C}$. Otherwise, compressor remains off to reduce the electricity cost.

The daily energy consumption of refrigerator is shown in Figure 10a. From $\left(t_{1} \rightarrow t_{4}\right)$, refrigerator's door is opened with a constant rate ( 2 times) based on which energy consumption is calculated. From $\left(t_{4} \rightarrow t_{5}\right),\left(t_{6} \rightarrow t_{7}\right),\left(t_{8} \rightarrow t_{11}\right),\left(t_{12} \rightarrow t_{19}\right)$ and $\left(t_{22} \rightarrow t_{23}\right)$, refrigerator's door is closed as shown via red dotted lines (Figure 10a). However, energy is still consumed because the compressor needs energy to keep inside temperature within desired limit (Figure 10b). However, the energy consumed in case of iPCT is less as compared to CPCT. Due to proper tuning of $\beta$ and $\gamma$ variables (Section 3.3), total energy consumption and electricity cost of refrigerator are comparatively less in case of iPCT. Using $\mathrm{PCCT}$, total energy consumption of refrigerator is reduced from $40.6864 \mathrm{kWh}$ to $34.53 \mathrm{kWh}$ which is $15.12 \%$ reduction. The daily electricity cost of refrigerator is 
reduced from $476 \$$ to $392 \$$, which is approximately $18 \%$ reduction. These results verify that under same conditions, the proposed algorithm efficiently reduces the daily electricity cost and maintains the internal temperature of refrigerator within desired range. The parameters and temperature set points used to model the optimization problem of a refrigerator are given in Table 5.

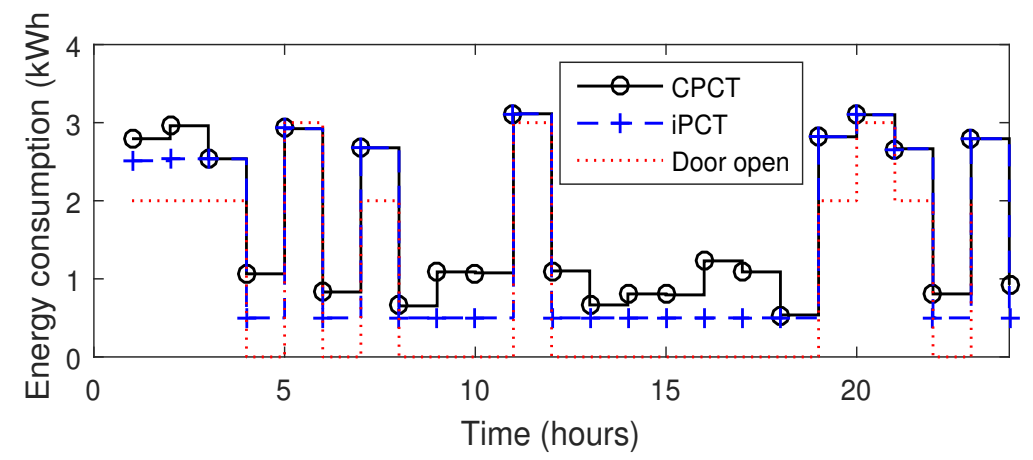

(a)

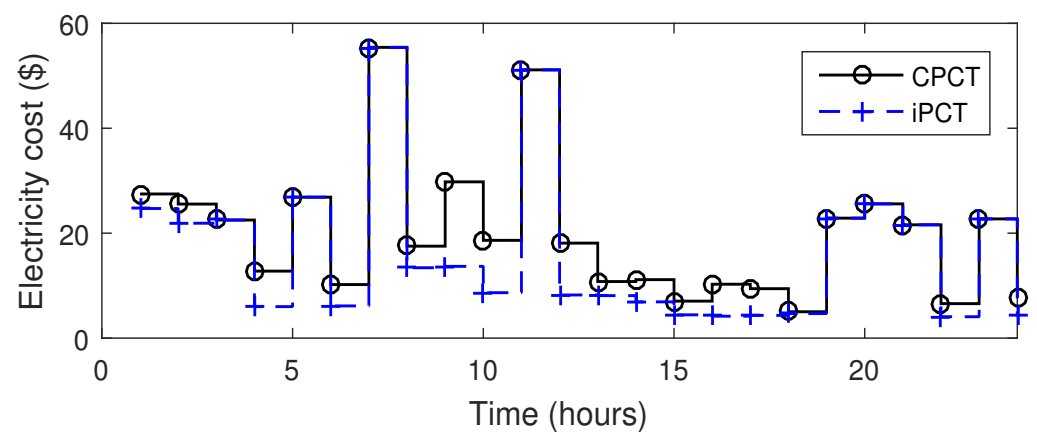

(b)

Figure 10. Comparison of energy consumption and electricity cost of refrigerator in unscheduled and unscheduled cases, (door open-total number of door open attempts): (a) energy consumption of refrigerator; and (b) daily electricity cost of refrigerator.

In order to visualize the impact of short time slot on energy management system and its stability, the proposed algorithms of HVAC and refrigerator are tested for $t=30 \mathrm{~min}$ time window. This section explains the energy consumption and electricity cost of HVAC and refrigerator in relation to three number of parameters: (i) total number of door open attempts; (ii) temperature variations; (iii) $t=30 \mathrm{~min}$ time slot. Considering CPCT, HVAC turns on based on temperature variations. While in case of $\mathrm{PCT}, \mathrm{HVAC}$ turns on based on temperature variations and total number of persons in the room. It is clear from Figure 11 that in case of iPCT, HVAC frequently turns on and off based on total number of persons present in the room. In Figure 12, total energy consumption and electricity cost of refrigerator is shown which is based upon total number of door open attempts (Section 3.3.1 (Refrigerator)). It is clear that during time slots $t_{8} \rightarrow t_{11}\left(t_{19} \rightarrow t_{21}\right)$, refrigerator's door is closed and resultant energy consumption is zero and vice verse. The total electricity cost savings of HVAC and refrigerator are $52 \%$ and $63.47 \%$, respectively. 

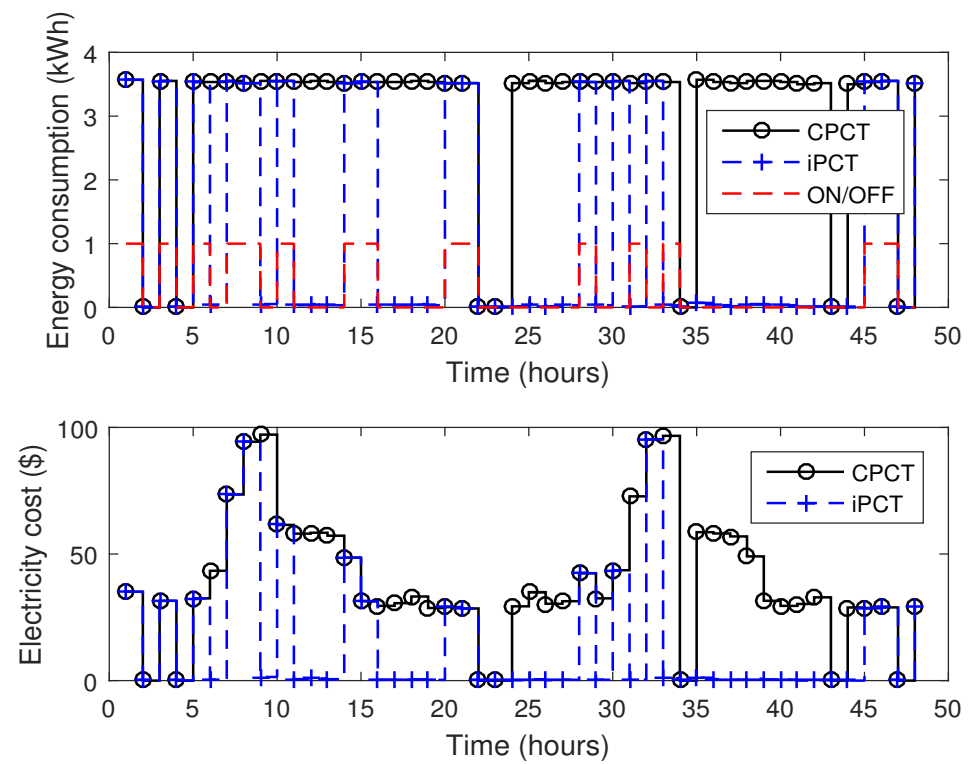

Figure 11. Total energy consumption and electricity cost of HVAC w.r.t total number of occupants $(t=30$ min time duration).
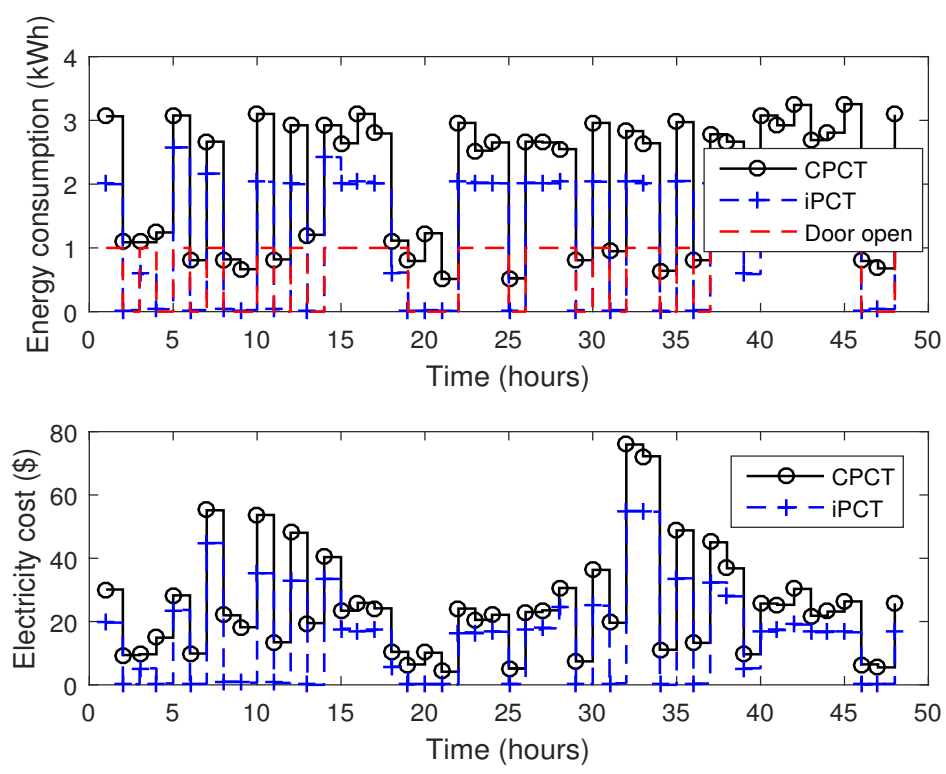

Figure 12. Total energy consumption and electricity cost of refrigerator w.r.t number of door opens $(t=30 \mathrm{~min}$ time duration).

Discussion: Figures 11 and 12 show that the proposed algorithms have the capability to manage the loads even if time window is less than $1 \mathrm{~h}$. Although, the energy management system works efficiently however there might be some complexities when using the small time window: (i) processing time can be increased; (ii) system complexities may increase because it is comparatively more complex to handle short time windows; (iii) it is very difficult for utilities to design real time DR programs based on small time slots. The reason behind this is the large amount of communication data exchanged between utilities and consumers. This is the main reason that most utilities are heavily relying on time of use (TOU) and day ahead pricing (DAP) schemes. Most importantly, the system with short time slot is efficient because $u a$ appliances can be turned off based on human occupancy. 
On the other hand, $u a$ appliances are scheduled on the basis of occupancy data. EMC turns on fans and lights if any person is present in the room. If $\delta=0$, it means that person is not present in the room and the associated appliance is off and vice versa. This mechanism reduces unnecessary energy consumption of $u a$ appliances and thus efficiently utilizes the energy. From Figure 13, it is clear that energy consumption of $u a$ appliances is almost half when occupancy and light sensors are not used. The energy consumption is reduced from $4.86 \mathrm{kWh}$ to $2.43 \mathrm{kWh}$ in a single day which is almost 50\% saving. The daily electricity cost of $u a$ appliances is shown in Figure 14 and the working of HVAC, refrigerator and $u a$ appliances is explained in Figure 15.

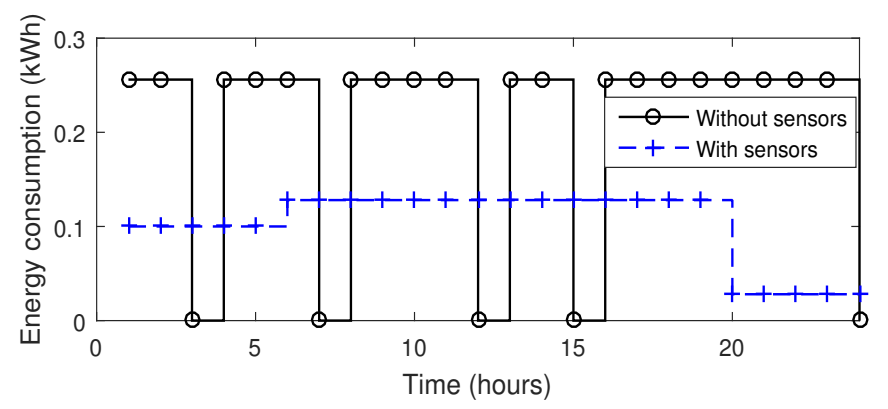

Figure 13. Energy consumption of $u a$ appliances.

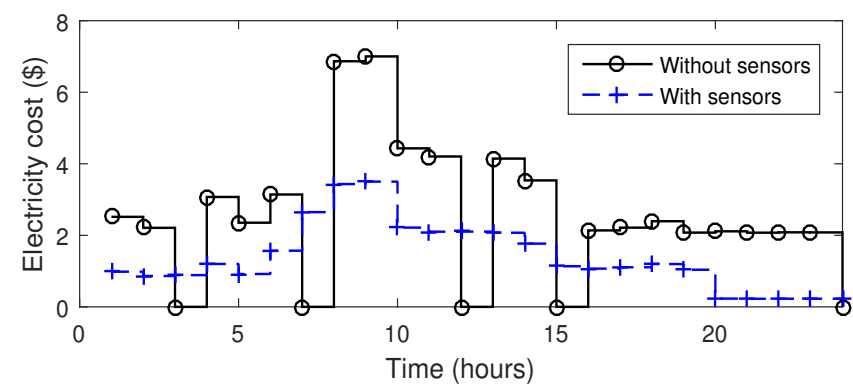

Figure 14. Daily electricity cost of $u a$ appliances.

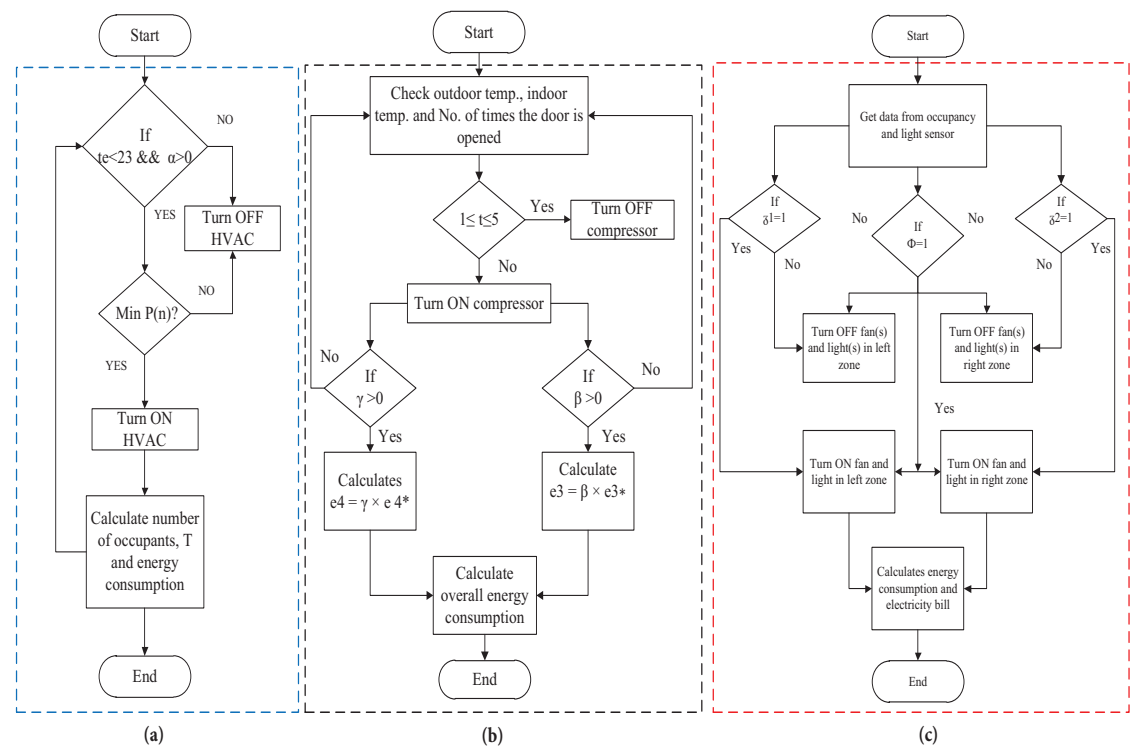

Figure 15. Energy management algorithms of (a) HVAC; (b) refrigerator and (c) ua appliances. 
The daily energy consumption and electricity cost of tc appliances (HVAC and refrigerator) and $u a$ appliances (fans and lights) are shown in Figure 16. In case of $u a, t c$ appliances, the daily electricity cost is reduced by $49.01 \%$ and $45.67 \%$, respectively. This is due to the fact that before turning $u a, t c$ appliances on, EMC checks the presence of person(s) in the room as described in (Sections 3.3.1 and 3.3.2). In traditional scheduling schemes, the working of HVAC is controlled on the basis of temperature difference only [22]. Furthermore, the electricity cost reduction of refrigerator is approximately $18 \%$ which shows that minimization of the opening duration of refrigerator's door leads to significant energy saving. It is also shown that our algorithm makes the CPCT more intelligent and operates at optimal cost while considering the HVAC constraints.

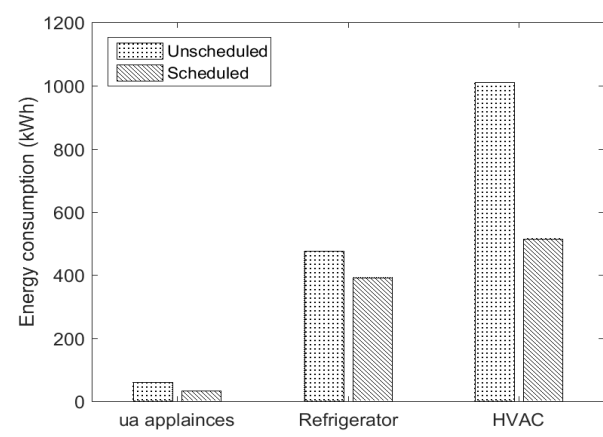

(a)

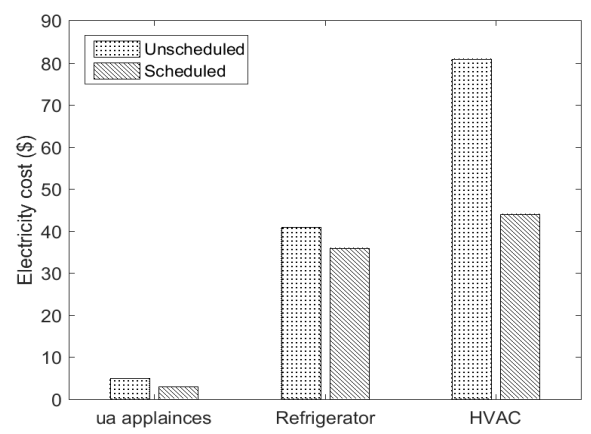

(b)

Figure 16. Comparison of total energy consumption and electricity cost of $u a$ and $t c$ appliances in unscheduled and scheduled cases: (a) energy consumption of $u a$ and $t c$ appliances; and (b) electricity cost of $u a$ and $t c$ appliances.

Figure 17 illustrates the energy consumption of $e l$ and $i e l$ and $r$ appliances. It is clear that the proposed algorithm efficiently schedules the appliances according to electricity prices and user preferences. The EMC schedules maximum appliances in low price hours $\left(h_{1} \rightarrow t_{8}\right)$ and $\left(h_{20} \rightarrow t_{24}\right)$, however it is also illustrated that some of the appliances are still running in critical time slots (i.e., $\left.t_{8} \rightarrow t_{12}\right)$. There are two main reasons behind this operation; (1) due to user comfort, because users do not want to compromise on their comfort; (2) due to total $l t_{i}$ constraint. One of the possible drawbacks of scheduling more appliances in low price hours is the possibility of high peaks on gird. In this situation, the proposed algorithm should be capable enough in handling high peak problem. So, we significantly avoid this problem by imposing knapsack capacity limit constraint Equation (9b). In general, the consumers are not the only stake holders in DSM programs, both utility and consumer can get equal benefits. This reduction in peak load demand decreases the PAR which improves the stability and the utilization factor of the grid.

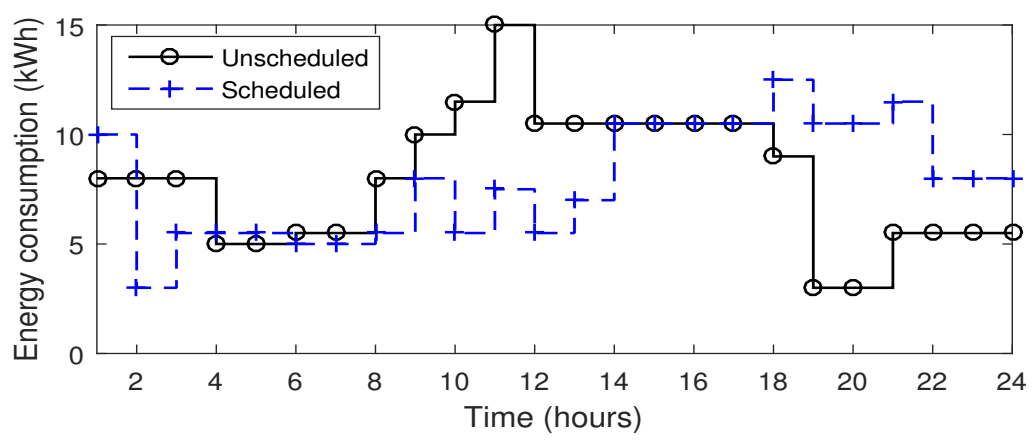

Figure 17. Daily energy consumption of $r, e l$ and iel appliances. 
In Figure 18, the hourly electricity cost of $e l$, iel and $r$ appliances is shown. It is clear from the figure that from time slot $\left(t_{8} \rightarrow t_{12}\right)$ there are high peak hours. The proposed algorithm efficiently shifts the controllable appliances in time slots $\left(t_{13} \rightarrow t_{24}\right)$ to reduce the electricity cost (due to constraints 8a-8e and 9a-9e). The $r$ appliances are still operated on diesel power generator (Section 3.1, their fuel cost is given in Table 7). This is because that the generation cost of microgrid is still less than the cost of utility. Therefore, it is feasible to operate $r$ appliances on generator to minimize the electricity cost and user frustration. The total electricity cost is high due to more energy consumed by iel appliances in these time slots. Another major reason is uninterruptible nature of these appliances. On the other hand, $e l$ appliances are interruptable and can be scheduled throughout the day (Section 3.3.3, Figure 19). Therefore, EMC schedules these appliances in low electricity time slots (i.e., $\left.\left(t_{0} \rightarrow t_{8}\right), t_{15} \rightarrow t_{24}\right)$. Regarding PAR, it is reduced by $22.77 \%$ using power capacity limit Equation (9b) which increases the efficiency and stability of grid. The peak load demand without the proposed algorithm is $16.5 \mathrm{~kW}$, whereas in case of proposed algorithm the peak load demand reduced to $14.5 \mathrm{~kW}$. This reduction in peak load demand reduces the total generation cost of peak power plants. Furthermore, the reduction in peak load demand and PAR reduces the line losses and improves the efficiency of grid. The steps involved in the controlling of $e l$ and $i e l$ appliances are shown in algorithm 2.

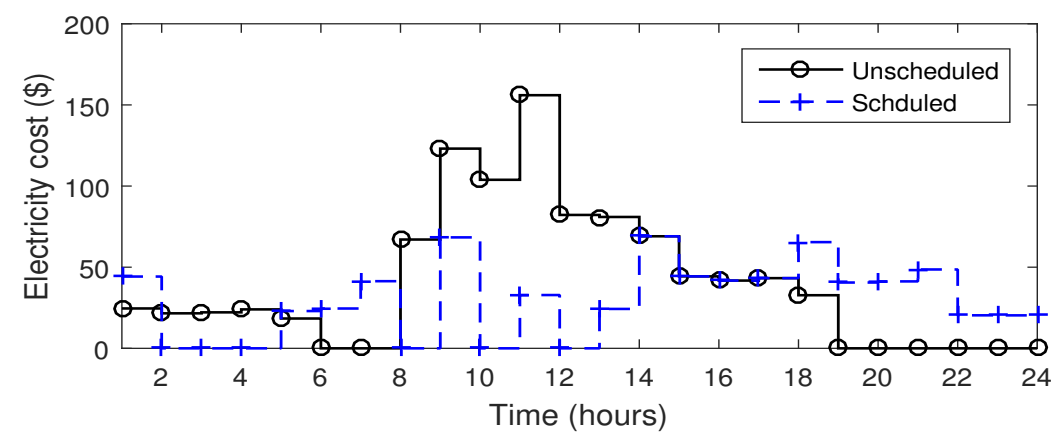

Figure 18. Hourly electricity cost of $r$, el and iel appliances.

Table 7. Generator output to serve $r$ appliances.

\begin{tabular}{cccccccc}
\hline Time (h) & ai & bi & ci & $P \min (\mathbf{k W})$ & $P \max (\mathbf{k W})$ & Pd & Cost (\$) \\
\hline $1-2$ & 0.001 & 0.007 & 7 & 2 & 10 & 5.5 & 7.07 \\
$3-4$ & 0.001 & 0.007 & 7 & 2 & 10 & 3 & 7.03 \\
5 & 0.001 & 0.007 & 7 & 2 & 10 & 5.5 & 7.07 \\
$6-7$ & 0.001 & 0.007 & 7 & 2 & 10 & 3 & 7.03 \\
$8-24$ & 0.001 & 0.007 & 7 & 2 & 10 & 5.5 & 7.07 \\
\hline
\end{tabular}

In Figure 20a, individual energy consumption patterns of $e l$ appliances which can be interrupted during their working cycles is shown. The el appliances have an interruptible nature and we can turn on/off where needed to reduce the electricity cost and PAR. When interrupted, these appliances wait for the next time slot where the electricity price is low. This may increase the waiting time of appliances which ultimately disturb user comfort (Section 3.2) due to comfort and cost tradeoff. Therefore, considering this tradeoff in mind, we design the algorithm which schedules the $e l$ appliances in such a way that their total average delay is not greater than 3 time slots (e.g., maximum waiting time flexibility is considered $3 \mathrm{~h}$ in our case). However, users have the flexibility to set the maximum waiting time according to their own desire. For example, washing machine and dishwasher can wait because these appliances are not directly related to user comfort (Section 3.2). To avoid the inconvenience, iel appliances are considered un-interruptible with high priority. These appliances remain on until the completion of complete cycles once turned on. The waiting time of 
these types of appliances is not more than $3 \mathrm{~h}$. It is clear from Figure $20 \mathrm{~b}$ that iel appliance is turned on at low electricity price hours. However, once turned on can not be interrupted during their duty cycle. Moreover, Figure 20b shows that the maximum delay of these appliances is equal to $3 \mathrm{~h}$ (high priority). Figure 19 shows the working mechanism of $r$, el and iel appliances.

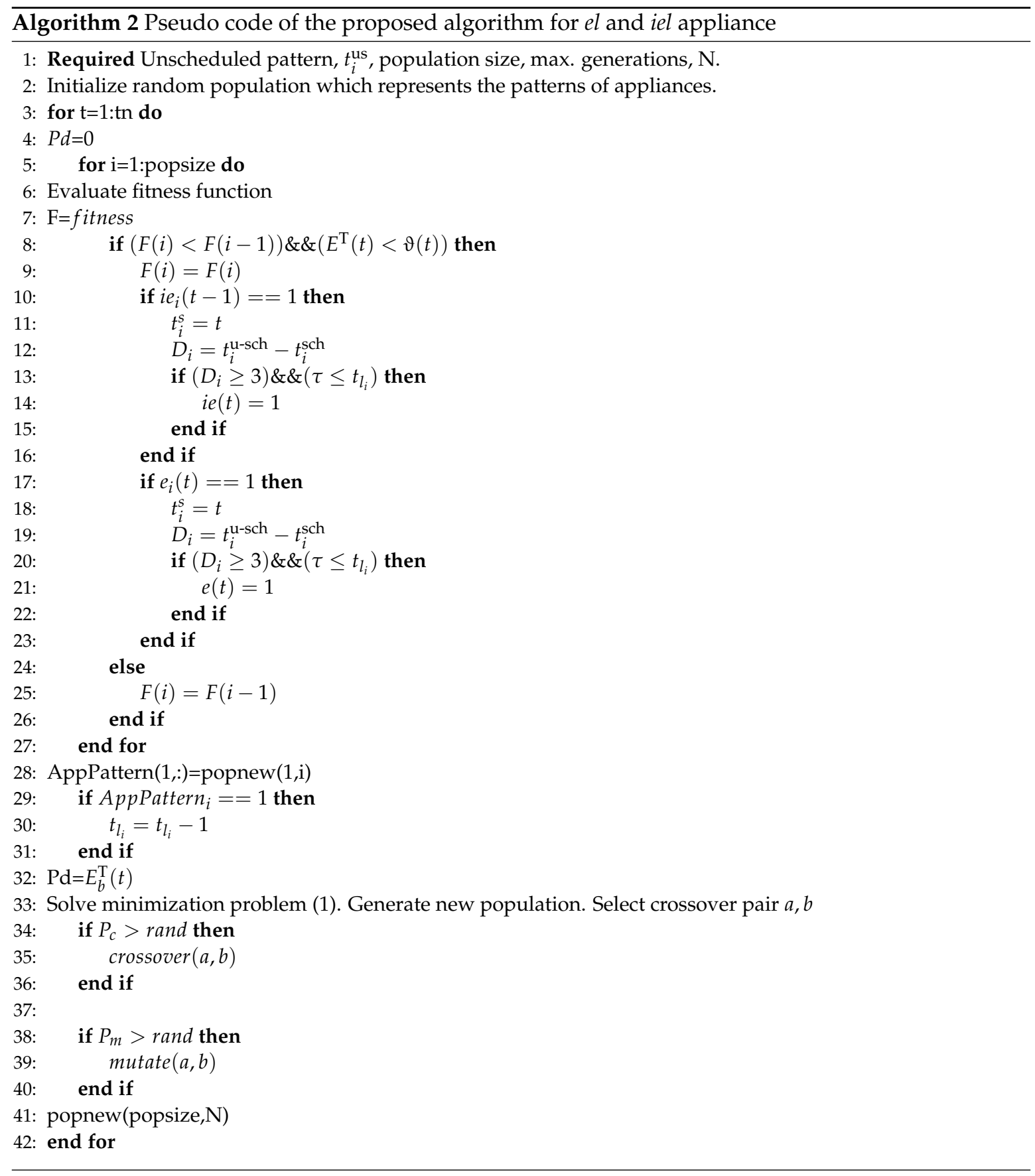

The average appliance waiting time of $r, e l$ and iel appliances is shown in Figure 21. $r$ appliances have zero waiting times because these appliances are served by local generation. Whereas, $\mathrm{el}$ appliances have average waiting time of $6 \mathrm{~h}$. From Figure 20, it is seen that in case of dishwasher the delay exceeds $3 \mathrm{~h}$.

Figure 22 shows user comfort in case of $e l$, iel and $r$ appliances. It is clear from the figure that frustration level of $r$ appliances is not shown because these appliances are turned on as per user demand. Moreover, $r$ appliances do not take part in DSM program and are not considered in 
optimization problem. Figure 22 shows that the user comfort level is less in case of iel appliances because the average waiting time of these appliances is less as compared to $e l$ appliances. The user comfort is $54 \%$ in case of $i e l$ appliances and $45 \%$ in case of $e l$ appliances. As discussed earlier that there is a tradeoff between user comfort and electricity cost reduction. If the users are interested in electricity cost reduction, then they have to compromise on comfort, otherwise, extra electricity cost must be paid. For instance, water pump is high power consumption device and its electricity cost depends on time of use. If these types of appliances are operated in non consecutive low pricing time slots, their energy consumption cost can be reduced. The proposed algorithm properly adjusts the working of iel appliances with minimum delay to maximize both comfort and electricity cost saving.

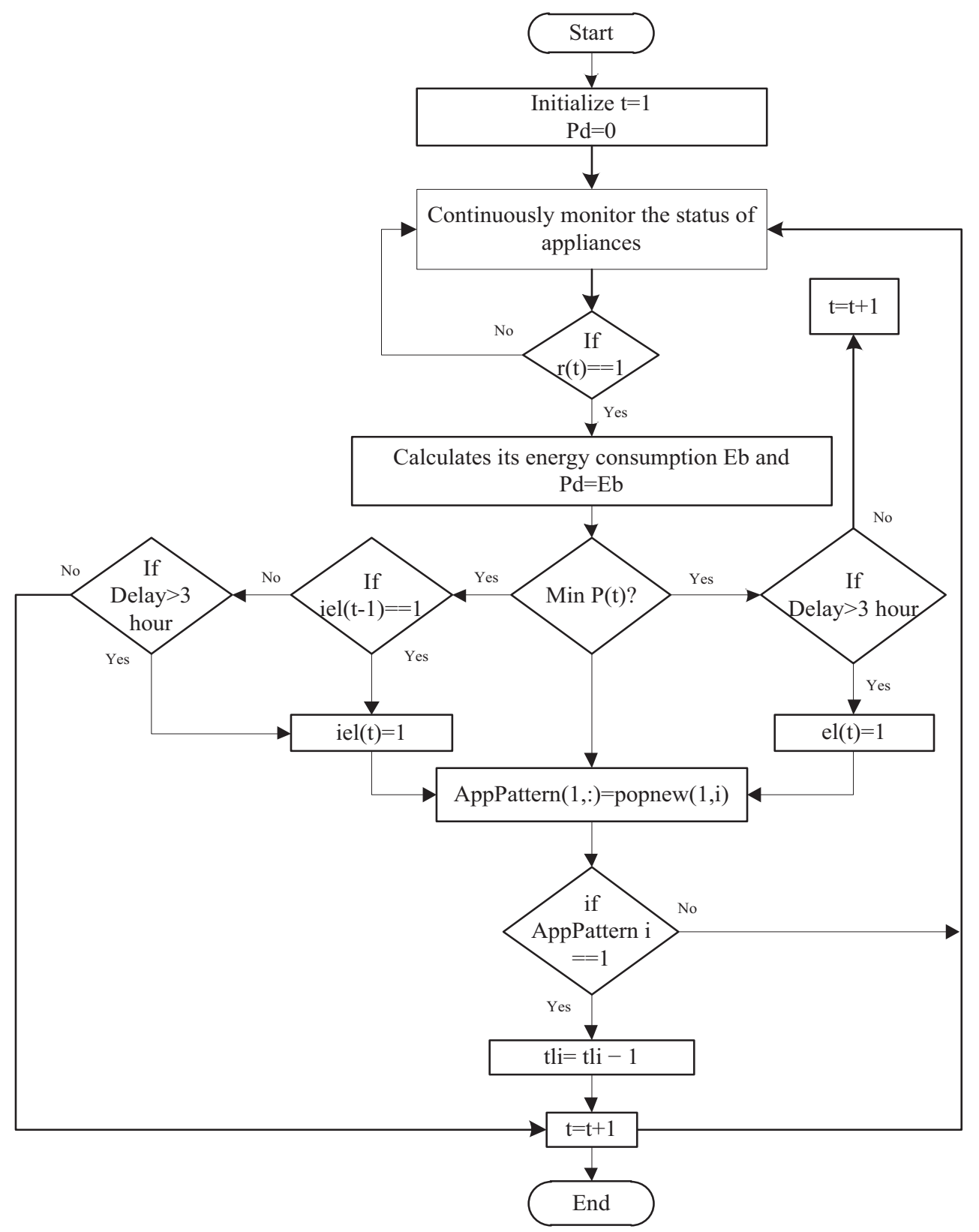

Figure 19. Energy management algorithm of $r$, el and iel appliances. 

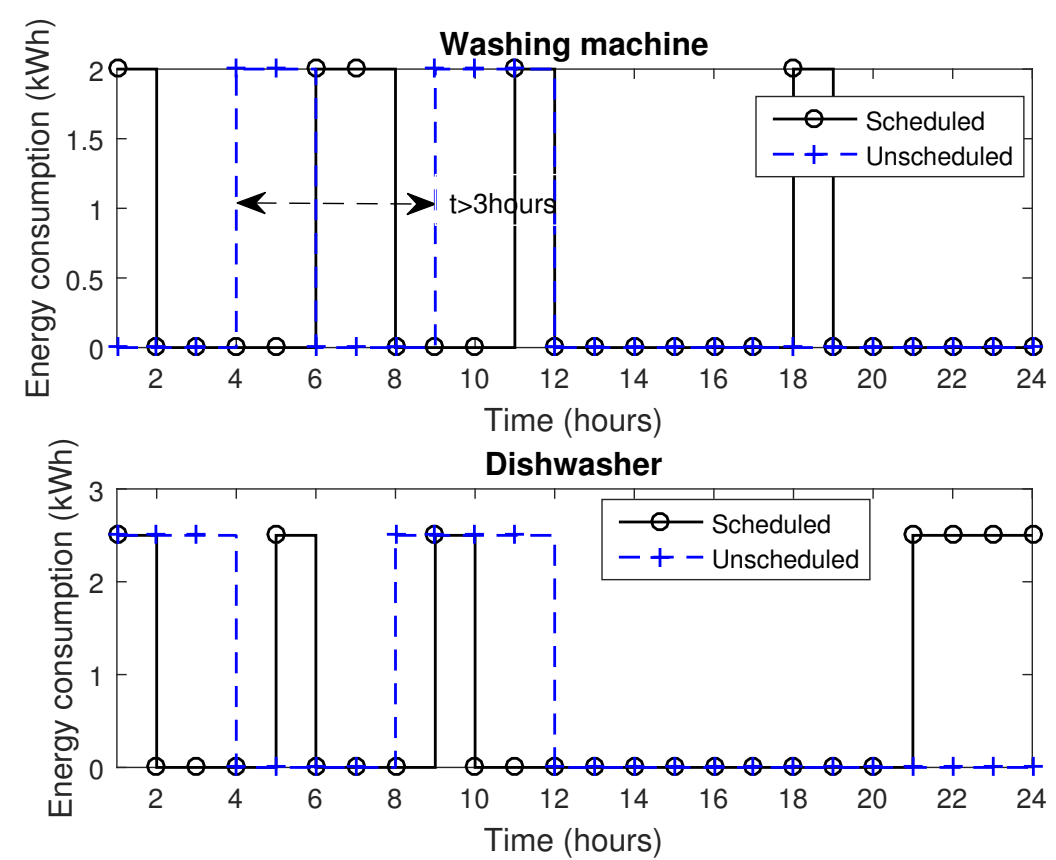

(a)
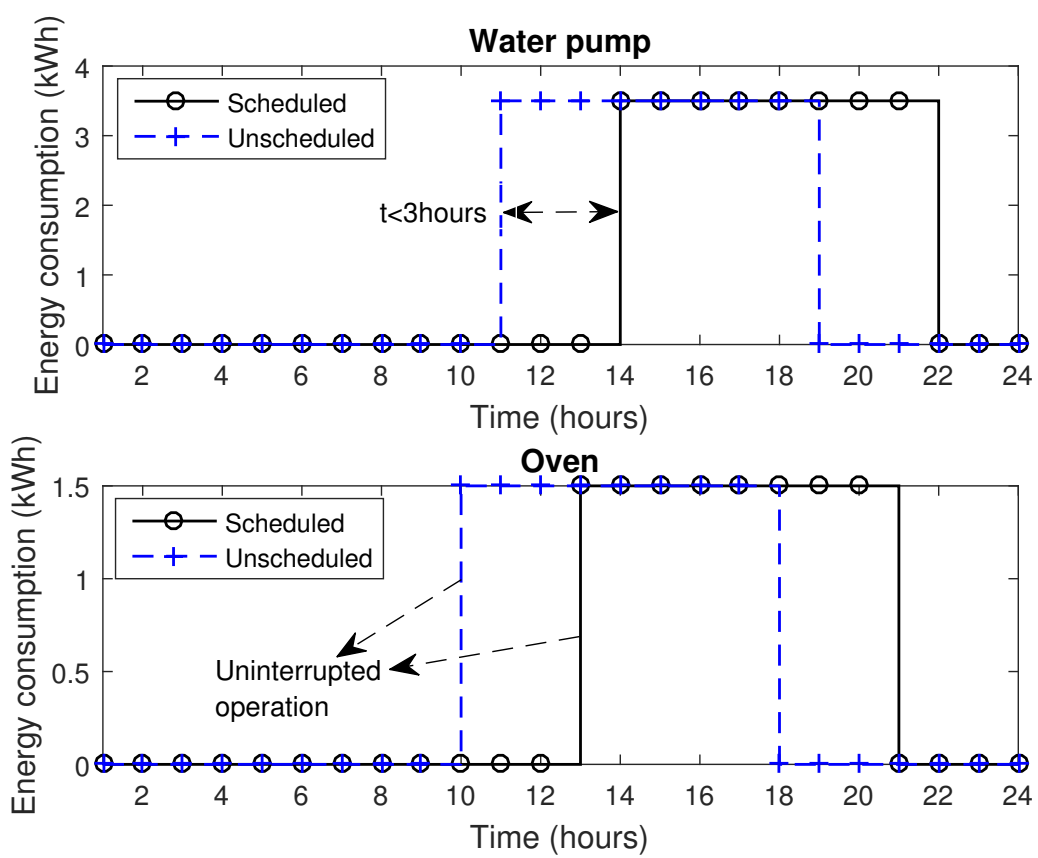

(b)

Figure 20. Appliance energy consumption and operating time slots in unscheduled and unscheduled cases: (a) energy consumption and optimal operating hours of el appliances; and (b) energy consumption and optimal operating hours of iel appliances. 


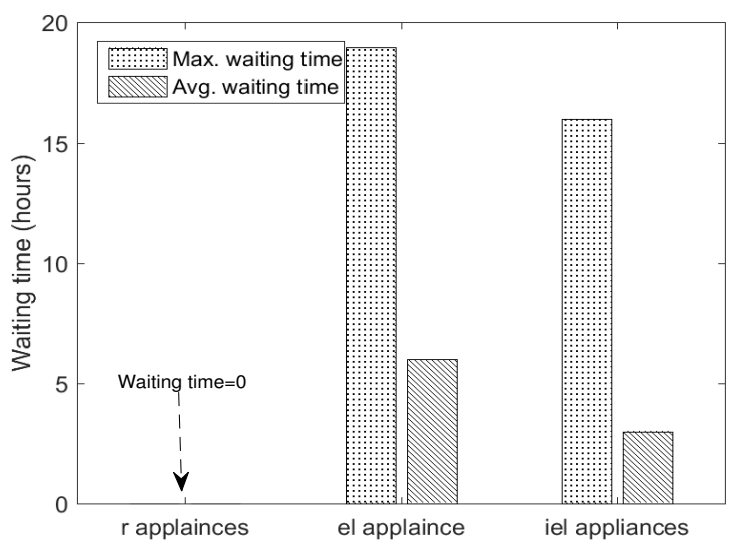

Figure 21. Average waiting time of $r, e l$ and iel appliances.

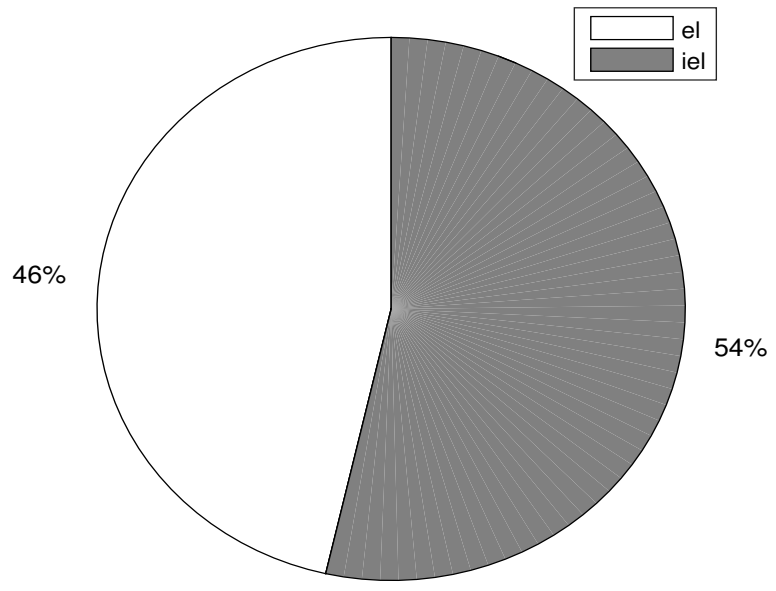

Figure 22. User comfort in case of $e l$ and iel appliances.

Remarks: in Table 8, we compare the performance of the proposed scheme with existing ones alongwith achievements and tradeoffs. From this table, it can be concluded that all the techniques are efficient in terms of cost reduction and load management. However, these techniques fail to manage the household load alongwith cost and energy consumption reductions. This is due to the inconsideration of user presence and thermal constrains which significantly minimize the tradeoff between user comfort and cost reduction. Moreover, user activities have direct impact on the energy consumption reduction. So, the proposed scheme gives best optimal results in terms of cost, energy consumption, and user discomfort reductions.

\section{Conclusion and Future Work}

In this work, we proposed algorithms for DSM based on GA to balance the residential load in a microgrid while maximizing user comfort and electricity cost savings. The proposed algorithms are flexible enough to achieve user comfort and reduce PAR as well. Furthermore, five types of appliances $(t c, u a, e l$, iel and $r$ ) are taken into consideration and mathematical models are proposed by considering thermal and comfort constraints. Based on extensive simulations, it is shown that the proposed algorithms efficiently manage the energy consumption by scheduling home appliances. The proposed algorithm reduced the electricity cost and PAR up to $22.63 \%$ and $22.77 \%$, respectively.

In future, we are interested in load and solar energy forecasting in any particular region based on which the energy management algorithm will be proposed to fulfill energy demand during high peak hours [36]. 
Table 8. Performance comparison of the proposed scheme with existing schemes, PP: person presence; AC: appliance categorization; PS: pricing scheme; ER : energy reduction; CR: cost reduction; DSM: demand side management; SG: smart grid; ILP: integer linear programming; HALM: home area load management; GA: genetic algorithm; PCT: programmable communication thermostat; iPCT: intelligent programmable communication thermostat; DR: demand response.

\begin{tabular}{|c|c|c|c|c|c|c|c|}
\hline Technique & Domain & Achievements & Limitations & $P P$ & ER & $\mathrm{AC}$ & CR \\
\hline Game theory [7] & $\begin{array}{l}\text { Appliance scheduling with } \\
\text { energy storage }\end{array}$ & $\begin{array}{l}\text { Minimize electricity cost, keep } \\
\text { user privacy high, storage optimization }\end{array}$ & $\begin{array}{l}\text { User comfort is not considered, focus is } \\
\text { towards cost reduction, environmental } \\
\text { conditions are not considered }\end{array}$ & $x$ & $x$ & $x$ & $\checkmark$ \\
\hline BPSO [2] & Appliance scheduling for DSM & $\begin{array}{l}\text { Minimize electricity cost, user comfort, } \\
\text { improved appliance utility }\end{array}$ & $\begin{array}{l}\text { Tradeoff between appliance utility and } \\
\text { user comfort }\end{array}$ & $\checkmark$ & $x$ & $\checkmark$ & $\checkmark$ \\
\hline Distributed algorithm [12] & Energy management in SG & $\begin{array}{l}\text { Reduce tradeoff between } \\
\text { exploiting renewable energy sources } \\
\text { and cost due to its volatility, } \\
\text { energy scheduling decision }\end{array}$ & User comfort is not considered & $x$ & $x$ & $x$ & $x$ \\
\hline Game theory [21] & Energy management in SG & $\begin{array}{l}\text { Reduce tradeoff between } \\
\text { exploiting renewable energy sources } \\
\text { and cost due to its volatility, } \\
\text { energy scheduling decision }\end{array}$ & User comfort is is neglected & $x$ & $x$ & $x$ & $x$ \\
\hline ILP [11] & HALM & Schedule peak load and optimal power & User comfort is not modeled & $x$ & $\checkmark$ & $\checkmark$ & $\checkmark$ \\
\hline $\begin{array}{l}\text { Proposed-GA, PCT, } \\
\text { iPCT }\end{array}$ & $\begin{array}{l}\text { Home appliance scheduling } \\
\text { with dynamic DR }\end{array}$ & $\begin{array}{l}\text { Minimize electricity cost, appliance avg. } \\
\text { waiting time, user frustration, fuel } \\
\text { cost of diesel generator, } \\
\text { energy consumption }\end{array}$ & $\begin{array}{l}\text { Tradeoff between comfort } \\
\text { and energy consumption }\end{array}$ & $\checkmark$ & $\checkmark$ & $\checkmark$ & $\checkmark$ \\
\hline
\end{tabular}


Acknowledgments: The authors would like to extend their sincere appreciation to the Deanship of Scientific Research at King Saud University for funding this Research group No. RG-037-1435.

Author Contributions: Muhammad Babar Rasheed, Muhammad Awais, and Zafar Iqbal did and wrote simulations. Zahoor Ali Khan, Umar Qasim, and Nabil Alrajeh classified the appliances and proposed the time slots. They performed mathematical formulations as well. Nadeem Javaid supervised overall work flow, set and refined the manuscript. Muhammad Babar Rasheed and Qaisar Javaid revised the manuscript according to the reviewers' comments.

Conflicts of Interest: The authors declare no conflict of interest.

\section{References}

1. Deploying a smarter grid through cable solutions and services, 2010. Available online: http://www.nexans.com/Corporate/2010/WHITE_PAPER_SMART_GRIDS_2010.pdf (accessed on 31 January 2016).

2. Yan, Y.; Qian, Y.; Sharif, H.; Tipper, D. A survey on smart grid communication infrastructures: Motivations, requirements and challenges. IEEE Commun. Surv. Tutor. 2013, 15, 5-20.

3. Ma, R.; Chen, H.H.; Huang, Y.R.; Meng, W. Smart grid communication: Its challenges and opportunities. IEEE Trans. Smart Grid 2013, 4, 36-46.

4. Ye, F.; Qian, Y.; Hu, R. Energy efficient self-sustaining wireless neighborhood area network design for smart grid. IEEE Trans. Smart Grid 2015, 6, 220-229.

5. Zhou, J.; Hu, R.; Qian, Y. Scalable distributed communication architectures to support advanced metering infrastructure in smart grid. IEEE Trans. Parallel Distrib. Syst. 2012, 23, 1632-1642.

6. Ipakchi, A.; Albuyeh, F. Grid of the future. IEEE Power Energy 2009, 7, 52-62.

7. Nguyen, H.K.; Song, J.B.; Han, Z. Distributed demand side management with energy storage in smart grid. IEEE Trans. Parallel Distrib. Syst. 2015, 26, 3346-3357.

8. Samadi, P.; Mohsenian-Rad, A.; Schober, R.; Wong, V.W.S.; Jatskevich, J. Optimal real-time pricing algorithm based on utility maximization for smart grid. In Proceedings of the 1st IEEE International Conference on Smart Grid Communications, Gaithersburg, MD, USA, 2010; pp. 415-420.

9. Mahmood, D.; Javaid, N.; Alrajeh, N.; Khan, Z.A.; Qasim, U.; Ahmed, I.; Ilahi, M. Realistic scheduling mechanism for smart homes. Energies 2016, 9, 202. doi:10.3390/en9030202.

10. Kantarci, M.E.; Mouftah, H.T. Wireless sensor networks for cost-efficient residential energy management in the smart grid. IEEE Trans. Smart Grid 2011, 2, 314-325.

11. Zhu, Z.; Tang, J.; Lambotharan, S.; Chin, W.H.; Fan, Z. An integer linear programming based optimization for home demand-side management in smart grid. In Proceedings of the IEEE PES Innovative Smart Grid Technologies, Washington, DC, USA, 16-20 January 2012; pp. 1-5.

12. Yuan, W.; Vincent, K.N.L.; Tsang, K.H.; Ping, Q.L.; Limin, M. Optimal energy scheduling for residential smart grid with centralized renewable energy source. IEEE Syst. J. 2014, 8, 562-576.

13. Agnetis, A.; de Pascale, G.; Detti, P.; Vicino, A. Load scheduling for household energy consumption optimization. IEEE Trans. Smart Grid 2013, 4, 2364-2373.

14. Yi, P.Z.; Dong, X.H.; Iwayemi, A.; Zhou, C.; Li, S.F. Real-time opportunistic scheduling for residential demand response. IEEE Trans. Smart Grid 2013, 4, 227-234.

15. Rasheed, M.B.; Javaid, N.; Ahmad, A.; Khan, Z.A.; Qasim, U.; Alrajeh, N. An efficient power scheduling scheme for residential load management in smart homes. Appl. Sci. 2015, 5, 1134-1163.

16. Faria, P.; Soares, J.; Vale, Z.; Morais, H.; Sousa, T. Modified particle swarm optimization applied to integrated demand response and DG resources scheduling. IEEE Trans. Smart Grid 2013, 4, 606-616.

17. Logenthiran, T.; Srinivasan, D.; Shun, T.Z. Demand side management in smart grid using heuristic optimization. IEEE Trans. Smart Grid 2012, 3, 1244-1252.

18. Chen, Z.; Wu, L.; Fu, Y. Real-time price-based demand response management for residential appliances via stochastic optimization and robust optimization. IEEE Trans. Smart Grid 2012, 3, 1822-1831.

19. Huang, S.; Xiao, J.; Pekny, J. F.; Reklaitis, G.V.; Liu, A.L. Quantifying system-level benefits from distributed solar and energy storage. J. Energy Eng. 2011, 138, 33-42.

20. Gatsis, N.; Giannakis, G.B. Residential load control: Distributed scheduling and convergence with lost AMI messages. IEEE Trans. Smart Grid 2012, 3, 770-786. 
21. Yaagoubi, N.; Mouftah, H.T. User-aware game theoretic approach for demand management. IEEE Trans. Smart Grid 2015, 6, 716-725.

22. Nguyen, D.T.; Le, L.B. Joint optimization of electric vehicle and home energy scheduling considering user comfort preference. IEEE Trans. Smart Grid 2014, 5, 188-199.

23. Nguyen, H.T.; Nguyen, D.; Le, L.B. Home energy management with generic thermal dynamics and user temperature preference. In Proceedings of the IEEE International Conference on Smart Grid Communications, Vancouver, BC, Canada, 21-24 October 2013; pp. 552-557.

24. Nguyen, D.T.; Le, L.B. Joint optimization of electric vehicle and home energy scheduling considering user comfort preference. IEEE Trans. Smart Grid 2014, 5, 188-199.

25. Wang, L.; Wang, Z.; Yang, R. Intelligent multiagent control system for energy and comfort management in smart and sustainable buildings. IEEE Trans. Smart Grid 2012, 3, 605-617.

26. Blerim, Q.; Mouftah, H.T. Observe, learn and adapt (OLA)-An algorithm for energy management in smart grid homes using wireless sensor and artificial intelligence. IEEE Trans. Smart Grid 2012, 3, 2262-2272.

27. Microgrids. Available online: https://building-microgrid.lbl.gov/about-microgrids (accessed on 31 January 2016).

28. Saadat, H. Power System Analysis; WCB/McGraw-Hill: Boston, MA, USA, 1999.

29. Lin, Y.H.; Tsai, M.S. An advanced home energy management system facilitated by nonintrusive load monitoring with automated multiobjective power scheduling. IEEE Trans. Smart Grid 2015, 6, 1839-1851.

30. Sumathi, S.; Paneerselvam, S. Computational Intelligence Paradigms: Theory and Applications Using MATLAB; CRC Press: Boca Raton, FL, USA, 2010.

31. D6T thermal sensor, high sensitivity enables detection of stationary human presence, 2012. Available online: http://www.digikey.com/en/product-highlight/o/omron/d6t-thermal-sensor (accessed on 22 June 2016).

32. Maharjan, I.K. Demand Side Management: Load Management, Load Profiling, Load Shifting, Residential and Industrial Consumer, Energy Audit, Reliability, Urban, Semi-Urban and Rural Setting; LAP (Lambert Acad. Publ.): Saar-Brucken, Germany, 2010.

33. How Much Power Do Your Appliances Use? Available online: http://www.wholesalesolar.com/solar-information/how-to-save-energy/power-table (accessed on 31 December 2015).

34. Reduce your electricity bills, 2014. Available online: http://www.dawn.com/news/1055880 (accessed on 12 February 2016).

35. Saidur, R.; Masjuki, H.H.; Mahlia, T.M.I.; Nasrudin, A.R. Factors affecting refrigerator-freezers energy consumption. ASEAN J. Sci. Technol. Dev. 2002, 19, 57-68.

36. Ahmad, A.; Javaid, N.; Alrajeh, N.; Khan, Z.A.; Qasim, U.; Khan, A. A Modified Feature Selection and Artificial Neural Network-Based Day-Ahead Load Forecasting Model for a Smart Grid. Appl. Sci. 2015, 5, 1756-1772.

(C) 2016 by the authors; licensee MDPI, Basel, Switzerland. This article is an open access article distributed under the terms and conditions of the Creative Commons Attribution (CC-BY) license (http://creativecommons.org/licenses/by/4.0/). 